P. Bullon', J.M. Morillo', M.C. RamirezTortosa ${ }^{2}$, J.L. Quiles ${ }^{3}$, H.N. Newman ${ }^{4}$, and M. Battino ${ }^{5 *}$

${ }^{1}$ Deptartment of Periodontology, Dental School, University of Sevilla, Spain; ${ }^{2}$ Institute of Nutrition and Food Technology, Department of Biochemistry and Molecular Biology II, University of Granada, Spain; ${ }^{3}$ Institute of Nutrition and Food Technology, Department of Physiology, University of Granada, Spain; ${ }^{4}$ University College London, UK; and ${ }^{5}$ Institute of Biochemistry, Faculty of Medicine, Università Politecnica delle Marche, Via Ranieri, 65, 60100 Ancona, Italy; *corresponding author, m.a.battino@univpm.it

J Dent Res 88(6):503-518, 2009

\section{ABSTRACT}

A review of pathological mechanisms that can explain the relationship between periodontitis and cardiovascular disease (CVD) is necessary to improve the management of both conditions. Metabolic syndrome is a combination of obesity, hypertension, impaired glucose tolerance or diabetes, hyperinsulinemia, and dyslipidemia. All these have been examined in recent years in terms of their relationship to periodontitis. Reviewed data indicate an association between some of them (body mass index, high-density lipoprotein-cholesterol [HDL-C], triglycerides, high blood pressure, among others) and periodontitis. Oxidative stress may act as a potential common link to explain relationships between each component of metabolic syndrome and periodontitis. Both conditions show increased serum levels of products derived from oxidative damage, with a pro-inflammatory state likely influencing each other bidirectionally. Adipocytokines might modulate the oxidant/anti-oxidant balance in this relationship.

KEY WORDS: metabolic syndrome, oxidative stress, periodontitis, hypertension, dyslipidemia, insulin resistance.

\section{DOI: $10.1177 / 0022034509337479$}

Received May 28, 2008; Last revision September 19, 2008; Accepted November 13, 2008

\title{
Metabolic Syndrome and Periodontitis: Is Oxidative Stress a Common Link?
}

\section{INTRODUCTION}

ardiovascular disease (CVD) is the major cause of death in western countries (World Health Organization [WHO], 2005). Metabolic syndrome (MetS) is a clinical entity that encompasses several risk factors for CVD (Semenkovich, 2006; N Pischon et al., 2008). MetS consists of a combination of impaired glucose regulation, abdominal obesity, dyslipidemia, and high blood pressure (Eckel et al., 2005). It is estimated that around a quarter of the world's adult population is affected by MetS (Cameron et al., 2004).

It is generally accepted that the origin of all those metabolic disorders is a "pro-inflammatory" state derived from excessive caloric intake and overnutrition, and, perhaps, other chronic inflammatory conditions (Dandona et al., 2004; Ghanim et al., 2004; Hotamisligil, 2006). This hypothesis states that this pro-inflammatory state, being characterized by an increase in inflammatory mediators such as tumor necrosis factor alpha (TNF- $\alpha$ ), induces insulin resistance, promoting further inflammation through an increased free fatty acid (FFA) concentration (essentially, derived from lipolysis) and a resultant interference with the anti-inflammatory effects of insulin. This pro-inflammatory state also leads to an increase in oxidative stress, with the potential to impair several crucial biological mechanisms (Tripathy et al., 2003; Dandona et al., 2004; Hansel et al., 2004). Therefore, insulin resistance could act as the common link among all the components of MetS (Dandona et al., 2002, 2004).

Oxidative stress is defined as a persistent imbalance between the production of highly reactive molecular species (e.g., reactive oxygen species [ROS], reactive nitrogen species [RNS]) and anti-oxidant defenses (Halliwell, 1991). There is an increase in ROS in the pre-diabetic stage, likely due to obesityrelated elevations of FFA, and several studies have shown that reversal of the imbalance between ROS and anti-oxidants improves insulin resistance in mice and humans (Ceriello and Motz, 2004; Wright et al., 2006).

Periodontitis is a generally chronic disorder characterized by the breakdown of the tooth-supporting tissues and the impaired host inflammatory immune response. This condition is due fundamentally to an ecological imbalance between the normal microbial biofilm on teeth and the host tissues (Newman, 1974). There is increasing evidence linking periodontitis to systemic diseases (Kuo et al., 2008), such as diabetes (Herring and Shah, 2006), rheumatoid arthritis (Pischon et al., 2008b), and, especially, CVD (Ford et al., 2007; Paquette et al., 2007; Fardi and Papadimitriou, 2008)-hence the search for factors that may explain such relationships. A potential factor which could increase insulin resistance is the production of oxidative stressenhancing ROS in affected periodontal tissues (Battino et al., 1999; Chapple and Matthews, 2007). 
Table 1. Current Definitions of Disorders with Impaired Glucose Regulation (World Health Organization, 1999; American Diabetes Association, 2005; Nichols et al., 2007)

\begin{tabular}{|c|c|c|}
\hline Type 2 Diabetes & $\begin{array}{l}\text { Impaired Glucose } \\
\text { Tolerance (IGT) }\end{array}$ & Impaired Fasting Glycemia (IFG) \\
\hline $\begin{array}{l}\text { - Single raised glucose reading } \\
\text { with symptoms, } \\
\text { or } \\
\text { - Raised values on } 2 \text { occasions, } \\
\text { of either fasting plasma } \\
\text { glucose } \geq 7.0 \mathrm{mmol} / \mathrm{L} \\
\text { (126 mg/dL) or with a } \\
\text { glucose tolerance test, } \\
2 \text { hrs after an oral dose of } 75 \\
\text { g, a plasma glucose } \geq \\
11.1 \mathrm{mmol} / \mathrm{L}(200 \mathrm{mg} / \mathrm{dL})\end{array}$ & $\begin{array}{l}\text { 2-hour glucose levels of } \\
\text { 140-199 mg per dL } \\
\text { (7.8-11.0 mmol/L) on a } \\
75-\mathrm{g} \text { oral glucose tolerance } \\
\text { test } \\
\text { - Fasting glucose may be } \\
\text { either normal or mildly } \\
\text { elevated }\end{array}$ & $\begin{array}{l}\text { Fasting glucose level > } 5.6 \\
\mathrm{mmol} / \mathrm{L}(100 \mathrm{mg} / \mathrm{dL}) \text { and } \\
<6.9 \mathrm{mmol} / \mathrm{L}(125 \mathrm{mg} / \mathrm{dL})\end{array}$ \\
\hline
\end{tabular}

Table 2. Definitions of Metabolic Syndrome by WHO and NCEP-ATP-III

\begin{tabular}{|c|c|}
\hline WHO (Alberti and Zimmet, 1998) & NCEP-ATP-III Criteria (NCEP-ATP, 2001) \\
\hline $\begin{array}{l}\text { Presence of DM, IGT, IFG, or insulin resistance, } \\
\text { and } 2 \text { of the following features: }\end{array}$ & At least three of the following: \\
\hline $\begin{array}{l}\text { - blood pressure } \geq 140 / 90 \mathrm{~mm} \mathrm{Hg} \text {; } \\
\text { - dyslipidemia, defined by TG } \geq 1.695 \mathrm{mmol} / \mathrm{L} \\
\text { and/or HDL-C } \leq 0.9 \mathrm{mmol} / \mathrm{L} \text { in males or } \leq 1.0 \\
\text { mmol/L in females; } \\
\text { - } \text { central obesity, defined by waist:hip ratio } \\
>0.90 \text { in males or }>0.85 \text { in females, and/or } \\
\mathrm{BMI}^{*}>30 \mathrm{~kg} / \mathrm{m}^{2} \text {; } \\
\text { - } \text { microalbuminuria, defined by a urinary albumin } \\
\text { excretion ratio } \geq 20 \mathrm{mg} / \mathrm{min} \text { or albumin: } \\
\text { creatinine ratio } \geq 30 \mathrm{mg} / \mathrm{g}\end{array}$ & $\begin{array}{l}\text { - central obesity, measured as waist } \\
\text { circumference } \geq 102 \mathrm{~cm} \text { in males or } \geq 88 \mathrm{~cm} \\
\text { in females; } \\
\text { - } \mathrm{TG} \geq 1.695 \mathrm{mmol} / \mathrm{L}(150 \mathrm{mg} / \mathrm{dL}) ; \\
\text { - } \mathrm{HDL}-\mathrm{C}<40 \mathrm{mg} / \mathrm{dL} \text { in males or }<50 \mathrm{mg} / \mathrm{dL} \\
\text { in females; } \\
\text { - blood pressure } \geq 130 / 85 \mathrm{mmHg} ; \\
\text { - fasting plasma glucose } \geq 6.1 \mathrm{mmol} / \mathrm{L} \\
\text { (110 mg/dL) }\end{array}$ \\
\hline
\end{tabular}

* BMI, body mass index; DM, diabetes mellitus; HDL-C, high-density lipoprotein-cholesterol; IFG, impaired fasting glycemia; IGT, impaired glucose tolerance; TG, triglycerides.

Therefore, our goal in this review is to analyze the published data to consider the hypothesis for a potential relationship between MetS and periodontitis, with oxidative stress acting as a putative link between both conditions.

\section{METABOLIC SYNDROME: CURRENT DEFINITIONS}

MetS as originally described (Reaven, 1988) is a combination of obesity, hypertension, impaired glucose tolerance or diabetes, hyperinsulinemia, and dyslipidemia (elevated triglycerides and decreased high-density lipoprotein-cholesterol [HDL-C] levels). These same features are also considered as risk factors for atherosclerosis, therefore leading to the deduction that MetS constitutes a risk for coronary heart disease (Ninomiya et al., 2004).

Insulin resistance is a condition in which normal amounts of insulin are inadequate to elicit a normal response from fat, muscle, and liver cells (Di Filippo et al., 2007). This condition leads to an eventual hyperglycemia which has systemic deleterious effects, mainly acting over the vasculature. The current definitions of the main disorders presenting with impaired glucose regulation are shown in Table 1.

There are currently several definitions in use to characterize MetS. The most frequently used are from the WHO, the US National Cholesterol Education Program Adult Treatment Panel III (NCEPATP-III, 2001) (Table 2), and the International Diabetes Federation (IDF, 2005) (Table 3). Other definitions come from the European Group for the Study of Insulin Resistance (Balkau et al., 2002) and the National Heart, Lung, and Blood Institute/American Heart Association (Grundy et al., 2004).

The rationale for the WHO definition was that a diabetic or prediabetic individual who developed certain CVD risk components should be considered as suffering from MetS, because this is a welldefined clinical entity. The WHO stated that each component conveyed a greater CVD risk, and their mixed occurrence increased the overall risk. Therefore, the reason for diagnosing MetS was to identify persons at undue risk of CVD (Reaven, 2006). Subsequently, the goal of the NCEP-ATP III was focused less on type 2 diabetes and more on CVD risk, and thus, an additional aim was to focus on primary prevention in persons with multiple risk factors. The NCEP-ATP III considered MetS to represent multiple, interrelated factors that raise CVD risk, and stated that the root causes were overweight or obesity, physical inactivity, and genetic factors (Reaven, 2006). The most recent characterization of MetS was the result of a consensus conference organized by the IDF. This definition gives more importance than others to ethnic differences in diagnostic criteria (IDF, 2005).

Nevertheless, there is currently some disagreement as to the precise definition of MetS (Reaven, 2005; Grundy, 2006). Some authors consider that a diagnosis of MetS as a defined entity is not clinically useful (Kahn et al., 2005; Kahn, 2007, 2008). They describe some weak features of this syndrome. With respect to definition, the criteria are ambiguous or incomplete, the rationale for thresholds in clinical or laboratory parameters is not clear, the inclusion of diabetes in the definition is questionable, and a clear basis for including or excluding other cardiovascular risk factors does not exist. Moreover, they argue that the cardiovascular risk associated with the syndrome seems to 
be no greater than the sum of its parts, and the treatment for MetS is not different from that for each of its components. These authors consider that the hypothesis of insulin resistance as a unifying cause is uncertain.

Therefore, it will be necessary to unify the diagnostic criteria to establish the actual prevalence and influence of this condition over local (e.g., periodontitis) and systemic diseases (e.g., CVD).

\section{OXIDATIVE STRESS AND
METABOLIC SYNDROME \\ OXIDATIVE STRESS AND
METABOLIC SYNDROME}

As previously mentioned, insulin resistance plays a key role in the pathophysiology of MetS. Several inflammatory mediators are involved in the pathogenesis of insulin resistance, with TNF- $\alpha$ having apparently the strongest effect (Tilg and Moschen, 2008). The most important tissues involved in the pathogenesis of this disorder are muscle and adipose tissue.

When caloric intake exceeds energy expenditure, the resultant substrate-induced increase in citric acid cycle activity generates an excess of ROS (Maddux et al., 2001). Oxidative stress alters the intracellular signaling pathway, inducing insulin resistance (Evans et al., 2003). Recently, a study with a murine model (Matsuzawa-Nagata et al., 2008) has shown that the pathways for ROS production and oxidative stress are up-regulated in both the liver and adipose tissue of mice fed a high-fat diet before the onset of insulin resistance. Moreover, the increased ROS production was previous to the TNF- $\alpha$ and FFA elevation in the plasma and liver. In agreement with this hypothesis, insulin resistance is associated with reduced intracellular anti-oxidant defense in humans (Bruce et al., 2003), and anti-oxidants improve insulin sensitivity (Paolisso and Giugliano, 1996; Ceriello, 2000).

There is a spectrum of potential molecular and cellular damage derived from ROS production. Lipoprotein modification takes place in the absence (glycation) and presence (glycoxidation) of oxygen (Baynes and Thorpe, 2000), and these modifications can alter their structure and function (Jenkins et al., 2004). These modified lipoproteins are formed through a non-enzymatic process in which sugars bind to free amino groups of the lipoprotein (Njoroge and Monnier, 1989; Basta et al., 2004).

Lipid peroxidation is the formation of lipid peroxides via an enzymatic and/or a non-enzymatic mechanism. ROS resulting from hyperglycemia are thought to contribute to the initiation of lipid peroxidation (Cosentino et al., 1997). Once formed, lipid peroxides undergo a series of complex reactions, ultimately binding chemically to proteins. Thus, lipoxidation is the covalent binding of products of lipid peroxidation reactions to proteins (Esterbauer et al., 1991, 1992; Spiteller, 1998; Jenkins et al., 2004).

Several studies have demonstrated a relationship between MetS and oxidative stress in humans. Thus, systemic oxidative stress is significantly higher in persons with MetS compared with non-obese normolipidemic individuals. Some HDL-C subfractions possess significantly lower specific anti-oxidative activity in affected persons than their counterparts in control individuals, and this attenuated anti-oxidative activity of HDL-C subfractions correlates with systemic oxidative stress and insulin resistance (Hansel et al., 2004). In agreement with these results, persons suffering from MetS have poor anti-oxidant status and significantly increased oxidative stress (serum lipid peroxide level), compared with those without MetS (Sharma et al., 2005). Moreover, obese adults with MetS have significantly higher plasma concentrations of oxidized-LDL-C, compared with obese adults without this condition (Van Guilder et al., 2006). By contrast, Sjogren et al. (2005) found no significant differences for plasma oxidized-LDL-C and urinary 8-iso-prostaglandin $\mathrm{F}_{2 \alpha}$ ( 8 -iso-PGF2 $\alpha$ ) between healthy and MetS men.

Advanced glycation end-products (AGE) are important markers for oxidative stress, and their endogenous secretory receptor (esRAGE) in plasma, as a soluble decoy receptor for AGE, is significantly and inversely correlated with components of the MetS, including body mass index, blood pressure, triglyceride, glycated hemoglobin (HbA1c), and an insulin resistance index (Koyama et al., 2005).

\section{METABOLIC SYNDROME AND PERIODONTITIS}

In spite of extensive clinical research on MetS, relatively little attention has been directed to its possible relationship to periodontitis. The available data come from epidemiological studies. In a group of 1315 affected individuals (30-92 yrs old), the prevalence of MetS was higher among individuals with advanced periodontitis $(66.7 \%)$ than in periodontally healthy individuals (48.8\%) (Borges et al., 2007). Analysis of data from 13,710 participants in the NHANES III (Third National Health and Nutrition Examination Survey) showed a direct relationship between periodontitis and the prevalence of MetS $37 \%$ in those with severe periodontitis $v s .18 \%$ in those with mild or no periodontitis), and, particularly, higher prevalence of obesity (48-54\% vs. 31\%), hypertension (51-56\% vs. 27\%), and high 
glucose levels (18-24\% vs. 8\%) were stated to be in the moderate to severe periodontitis group compared with the mild periodontitis or periodontally healthy group (D'Aiuto et al., 2008). In another paper (Shimazaki et al., 2007), larger waist circumference, decreased HDL-C levels, and higher fasting glucose levels were associated with significantly higher odds ratios (OR) for greater pocket depth values $(1.8,2.2$, and 2.2, respectively) in 584 Japanese women.

To study the pathological aspects behind this epidemiological relationship, one must analyze the different aspects of MetS in relation to periodontitis.

\section{Impaired Glucose Regulation and Periodontitis}

There are many papers on the relationship between diabetes and periodontal disease (Liu et al., 2006; Graves et al., 2007; Mealey and Ocampo, 2007; Nishimura et al., 2007; Preshaw et al., 2007), and evidence of the relationship between a major marker of diabetes, glycated hemoglobin ( $\mathrm{HbA} 1 \mathrm{c})$, and periodontal parameters (Grossi et al., 1997; Iwamoto et al., 2001; Stewart et al., 2001; Tsai et al., 2002; Navarro- Sanchez et al., 2007) exists.

As previously mentioned, one of the main common factors between both diseases is oxidative stress. The main studies relating oxidative stress as a common feature in periodontitis and diabetes are shown in Table 4. With respect to neutrophil function, there is no remarkable variation in oxidative burst and chemotaxis (De Toni et al., 1997; Christgau et al., 1998; Fontana et al., 1999). However, a decreased superoxide-dismutase (SOD) activity, an anti-oxidant enzyme, in gingival tissue from persons with periodontitis but without diabetes (Akalin et al., 2008), compared with those with periodontitis and diabetes, might be explained by a potential compensating mechanism in this enzymatic system derived from hyperglycemia. Individuals with periodontitis and diabetes also show decreased activity of one pro-oxidant enzyme, myeloperoxidase, in gingival crevicular fluid (GCF), compared with those without diabetes (Gonçalves et al., 2008). Analysis of these data suggests a dysregulation in oxidative balance derived from neutrophil leukocytes, with a concomitant influence by both conditions. Nevertheless, a critical point in the assessment of neutrophil function is the diversity and variability in assays, so concluding data in this respect are difficult to obtain.

With respect to substances derived from oxidative damage, a correlation exists between plasma lipid peroxidation and periodontal parameters in diabetic individuals (Collin et al., 2000; Sonoki et al., 2006). Moreover, there is increasing evidence of the deleterious effect of AGE on the pathogenesis (Schmidt et al., 1996) and progression of periodontitis (Takeda et al., 2006), and this effect could be mediated through the highly expressed RAGE in periodontal tissues (Katz et al., 2005). This last study found a similar expression of RAGE between diabetic and non-diabetic individuals by immunohistochemistry, but a higher mRNA level in diabetic individuals. A possible alternative splicing of mRNA from this molecule may explain this finding, and could elucidate differences in this regard between periodontal tissues and other body structures.

\section{Dyslipidemia and Periodontitis}

In recent years, several papers have considered the possible relationships between periodontitis and lipid parameters (Table 5). In general, although differences among studies exist, there is an association between increased LDL-C and triglyceride levels, as well as decreased HDL-C levels, and periodontitis. It is important to note the potential importance of oxidative stress in this relationship, because of the correlation existing between clinical periodontal status and plasma levels of anti-oxidized-LDL-C antibodies (Montebugnoli et al., 2004). Nevertheless, another report found no association between oxidized LDL-C and periodontal status (Türkoğlu et al., 2008). More research focused on the relationship between lipid peroxidation markers and periodontitis is warranted.

\section{Hypertension and Periodontitis}

The first report (Perlstein and Bissada, 1977) relating high blood pressure with periodontitis in animals found hyperplasia/ hypertrophy in the blood vessel walls from a chronically irritated gingiva in hypertensive and obese-hypertensive rats. However, hypertension alone was not a significant factor. In contrast to this, another report (Leite et al., 2005), in which an experimental ligature-induced periodontitis model was used in spontaneously hypertensive and normotensive rats, found that the ligated sides in the experimental group showed moderate to severe collagen degradation in the alveolar process, compared with mild degradation in controls.

The main studies relating hypertension and periodontitis in humans are shown in Table 6. In general, there is increasing evidence of a relationship between high blood pressure and more severe periodontal parameters, in such a way that individuals with hypertension show a poorer periodontal state (Wakai et al., 1999; Khader et al., 2003; Al-Emadi et al., 2006; Gołebiewska et al., 2006; Holmlund et al., 2006; SalcedoRocha et al., 2006; Engström et al., 2007), and periodontitis can negatively influence certain features of hypertension, such as an increase in the left ventricular mass (Angeli et al., 2003; Valentaviciene et al., 2006).

\section{Obesity and Periodontitis}

Recently, two exhaustive reviews have presented the main studies relating obesity and periodontitis (Pischon et al., 2007; Saito and Shimazaki, 2007), and readers are referred to these valuable sources. Briefly, both papers corroborate an influence of body mass index (BMI) and other anthropometric parameters on periodontitis, although the authors consider several limitations in previous studies. In the future, it would be necessary to use samples stratified by age, gender, and ethnicity, as well as by number of remaining teeth, to confirm those results, and in addition to design longitudinal studies to verify a potential causal relationship. Both reviews also consider adipocytokines as a potential link between obesity and periodontitis. In this respect, several reports relate leptin, adiponectin, and resistin with periodontitis, and are discussed below. 
Table 4. Main Studies about Oxidative Stress Parameters in Diabetes Mellitus and Periodontitis in Humans

\begin{tabular}{|c|c|c|c|c|c|c|}
\hline Reference & Study Population & Country & $\begin{array}{c}\text { Age } \\
\text { Range (yrs) }\end{array}$ & $\begin{array}{l}\text { Assessment of } \\
\text { Periodontitis }\end{array}$ & Oxidative Parameters & Major Results \\
\hline $\begin{array}{l}\text { Akalin } \\
\quad \text { et al., } 2008\end{array}$ & $\begin{array}{l}17 \text { type-2 DM persons } \\
\text { with CP (DMCP } \\
\text { group) } \\
18 \text { type-2 DM PH } \\
\text { persons (DMPH } \\
\text { group) } \\
17 \text { persons with CP } \\
\text { (CP group) } \\
17 \mathrm{PH} \text { control } \\
\text { individuals (PH } \\
\text { group) }\end{array}$ & Turkey & $29-68$ & $\begin{aligned}- & \geq 30 \% \mathrm{ABL}^{*} \text { and } \\
& \geq 3 \text { teeth with } \mathrm{PD} \\
& \geq 5 \mathrm{~mm}\end{aligned}$ & $\begin{array}{l}\text { - } \text { Gingival SOD } \\
\text { (measured as U/ } \\
\text { mg-protein) }\end{array}$ & $\begin{array}{l}\text { - Gingival SOD activity } \\
\text { was decreasing in this } \\
\text { order: } \mathrm{DMPH}>\mathrm{PH}> \\
\mathrm{DMCP}>\mathrm{CP} \text {, with } \\
\text { significant difference } \\
\text { between DMCP and } \\
\mathrm{DMPH} \text {, and between } \mathrm{CP} \\
\text { and } \mathrm{DMCP} \text { and } \mathrm{PH} \\
\text { - Inverse correlation } \\
\text { between gingival SOD } \\
\text { activity and } \mathrm{PD}, \mathrm{AL} \text {, } \\
\mathrm{Gl}, \mathrm{PI}\end{array}$ \\
\hline $\begin{array}{l}\text { Christgau } \\
\text { et al., } 1998\end{array}$ & $\begin{array}{l}\text { Persons with moderate } \\
\text { to severe CP: } \\
20 \text { DM persons } \\
20 \text { SH control } \\
\text { individuals }\end{array}$ & Germany & $30-67$ & $\begin{array}{l}-\quad \geq 16 \text { remaining } \\
\text { teeth } \\
\text { - } \geq 6 \text { teeth with PD } \\
\geq 4 \mathrm{~mm}\end{array}$ & $\begin{array}{l}\text { - Oxidative burst } \\
\text { response of PMN to } \\
\text { TNF- } \alpha \text { and FMLP, } \\
\text { before and after non- } \\
\text { surgical periodontal } \\
\text { therapy }\end{array}$ & $\begin{array}{l}\text { - No differences between } \\
\text { both groups in relation } \\
\text { to oxidative burst } \\
\text { response. } \\
\text { - > oxidative burst } \\
\text { response after } \\
\text { periodontal therapy, in } \\
\text { both groups }\end{array}$ \\
\hline $\begin{array}{l}\text { Collin et al., } \\
2000\end{array}$ & $\begin{array}{l}45 \text { type- } 2 \text { DM persons } \\
77 \text { control persons }\end{array}$ & Finland & $45-64$ & $\begin{array}{l}\text { - } \geq 1 \text { periodontal } \\
\text { pockets }>4 \mathrm{~mm}\end{array}$ & $\begin{array}{l}\text { - Salivary MMP-8 and } \\
\text { MMP-9 activities } \\
\text { - Plasma lipid } \\
\text { peroxidation }\end{array}$ & $\begin{array}{l}\text { - Poor metabolic control in } \\
\text { DM group was } \\
\text { associated with > } \\
\text { salivary MMP-8 but < } \\
\text { plasma lipid } \\
\text { peroxidation }\end{array}$ \\
\hline $\begin{array}{l}\text { Sonoki et al., } \\
2006\end{array}$ & $\begin{array}{l}\text { Persons with } \\
\text { periodontitis: } \\
5 \text { type-2 DM persons } \\
6 \text { SH persons }\end{array}$ & Japan & $>40$ & $\begin{array}{l}\text { - } A B L>50 \% \text { for } \geq \\
1 \text { tooth and } P D> \\
4 \mathrm{~mm} \text { for } \geq 1 \\
\text { tooth }\end{array}$ & $\begin{array}{l}\text { - } \text { Plasma lipid } \\
\text { peroxidation (LPO) } \\
\text { - Serum anti- } \\
\text { malondialdeyde- } \\
\text { modified LDL-C (anti- } \\
\text { MDA-LDL-C) }\end{array}$ & $\begin{array}{l}\text { - }>\text { LPO in DM compared } \\
\text { with control persons, but } \\
\text { no differences in anti- } \\
\text { MDA-LDL-C between } \\
\text { both groups } \\
\text { - } \quad \text { LPO in DM persons } \\
\text { after periodontal } \\
\text { therapy, but not in } \\
\text { control individuals }\end{array}$ \\
\hline
\end{tabular}


Table 4. (continued)

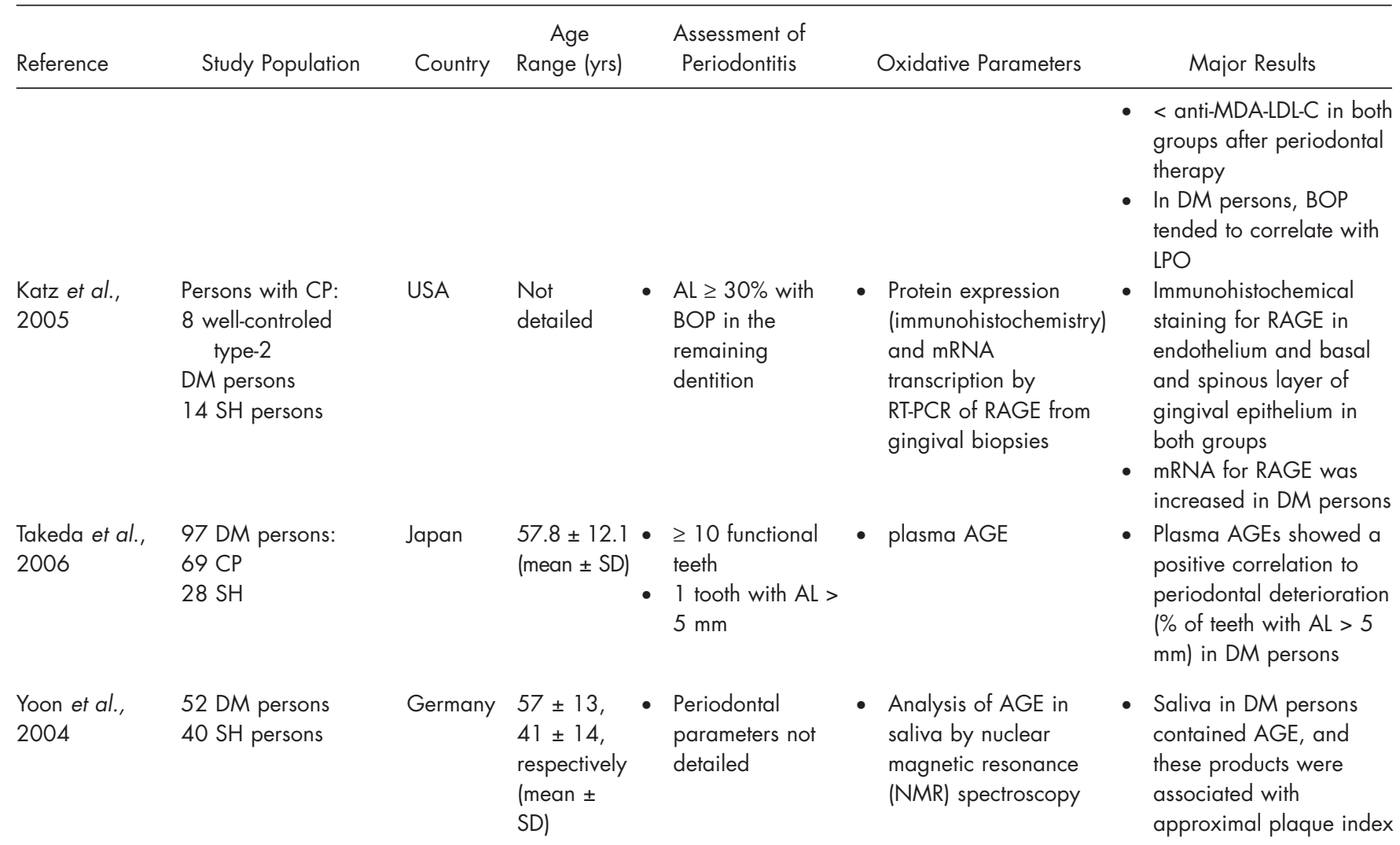

* $A B L$, alveolar bone loss; $A G E$, advanced glycation end-products; $A L$, attachment loss; $B O P$, bleeding on probing; periodontitis, CP, chronic periodontitis; DM, diabetes mellitus; FMLP, formyl-methyonyl-leucyl-phenylalanine; GCF, gingival crevicular fluid; GI, gingival index; LPO, lipid peroxidation; PD, probing depth; PH, periodontally healthy; PI, plaque index; RAGE, receptor for AGE; RT-PCR, reverse-transcription polymerase chain-reaction; SD, standard deviation; SH, systemically healthy; SOD, superoxide-dismutase; TNF- $\alpha$, tumor necrosis factor alpha.

\section{ADIPOCYTOKINES AND PERIODONTITIS}

The role of adipose tissue in the regulation of glucose homeostasis through insulin action has led to its consideration as a new endocrine organ. Adipocytes secrete a diversity of molecules, currently named adipocytokines, which influence metabolic and immune functions. Leptin and adiponectin are the most studied. Another molecule weakly related to adipocytes in humans, but very important in the inflammatory response and insulin resistance, is resistin (Juge-Aubry et al., 2005; Koerner et al., 2005; Rosen and Spiegelman, 2006).

\section{Leptin and Periodontitis}

Leptin negatively regulates the appetite and weight, mainly through a central mechanism involving the hypothalamus. It also can interact with other hormones, such as insulin (Margetic et al., 2002; Guzik et al., 2006). Obesity appears to be a condition of relative leptin resistance, with an elevated circulating level of leptin reported due to an enlarged fat mass (El-Haschimi et al., 2000). Moreover, there is some evidence that leptin is involved in the pathogenesis of atherosclerotic vascular disease (Wolk et al., 2004).
A decreasing leptin level in GCF and gingival tissue is associated with a more deteriorated periodontal status (Johnson and Serio, 2001), and smokers also show reduced GCF leptin levels (Bozkurt et al., 2006), suggesting a protective role of leptin for the periodontium. The relationship between GCF and serum levels of leptin has been recently reported (Karthikeyan and Pradeep, 2007a,b). GCF leptin levels are proportional to BMI. In periodontitis, there is a significant negative correlation between GCF and serum leptin concentration, and these changes are significantly associated with increasing clinical attachment loss. The two possible mechanisms to explain this inverse correlation are shown in Fig. 1. By contrast, a study of periodontitis and MetS (Borges et al., 2007) found a higher serum leptin level in healthy and chronic gingivitis individuals than in those with initial/moderate or advanced periodontitis. It is difficult to draw conclusions from this disagreement, because this latter study used pooled data from healthy and MetS individuals.

\section{Adiponectin and Periodontitis}

Adiponectin levels remain relatively constant in normal circumstances (Trujillo and Scherer, 2005), but are decreased in 
Table 5. Main Studies about Plasma Lipid Parameters and Periodontitis in Humans

\begin{tabular}{|c|c|c|c|c|c|c|}
\hline Reference & Study Participants & Country & $\begin{array}{l}\text { Age Range } \\
\text { (yrs) }\end{array}$ & $\begin{array}{l}\text { Assessment of } \\
\text { Periodontitis }\end{array}$ & $\begin{array}{l}\text { Serum Lipid } \\
\text { Parameters }\end{array}$ & Major Results \\
\hline $\begin{array}{l}\text { Cutler } \\
\text { et al., } 1999\end{array}$ & $\begin{array}{l}6 \mathrm{SH} \mathrm{PH} \\
7 \mathrm{SH} \mathrm{CP} \\
6 \text { well-controlled } \\
\mathrm{DM} \text { and } \mathrm{PH} \\
5 \text { well-controlled } \\
\text { and } \mathrm{CP} \\
5 \text { poorly-controlled } \\
\text { DM and PH } \\
6 \text { poorly-controlled } \\
\mathrm{DM} \text { and } \mathrm{CP}\end{array}$ & USA & $\begin{array}{l}28.2 \pm 4.6 \\
42.9 \pm 11.9 \\
52 \pm 6.4 \\
65.8 \pm 6.3 \\
45 \pm 5.3 \\
42.5 \pm 13.6 \\
\text { respectively } \\
\text { (mean } \pm \text { SD) }\end{array}$ & $\begin{array}{l}\text { - } \geq 4 \text { periodontal } \\
\text { pockets with } \\
\geq 6 \mathrm{~mm} \text { with } \mathrm{BOP} \\
\text { and radiographic } \\
\text { evidence of } A B L^{*} \\
>50 \%\end{array}$ & $\begin{array}{l}\text { - } \text { Total cholesterol } \\
\text { - HDL-C } \\
\text { - } \text { LDL-C } \\
\text { - } \text { TG }\end{array}$ & $\begin{array}{l}\text { - TG levels tended to be } \\
\text { higher in those groups } \\
\text { without CP }\end{array}$ \\
\hline $\begin{array}{l}\text { Wakai } \\
\text { et al., } 1999\end{array}$ & $\begin{array}{l}517 \text { males } \\
113 \text { females }\end{array}$ & Japan & $23-83$ & - CPITN & $\begin{array}{l}\text { - Total cholesterol } \\
\text { - HDL-C } \\
\text { - } \text { LDL-C } \\
\text { - } \quad \text { TG }\end{array}$ & $\begin{array}{l}\text { - }>\text { HDL-C level was } \\
\text { associared with < CPITN }\end{array}$ \\
\hline $\begin{array}{l}\text { Lösche } \\
\text { et al., } 2000\end{array}$ & $\begin{array}{l}39 \text { moderate } \mathrm{CP} \\
40 \mathrm{PH} \text { control } \\
\text { individuals }\end{array}$ & Germany & $50-60$ & $\begin{array}{l}\text { - }>3 \text { pockets with a } \\
P D \geq 4 \mathrm{~mm}\end{array}$ & $\begin{array}{ll}\text { - } & \text { Total cholesterol } \\
\text { - } & \text { LDL-C } \\
\text { - } & \text { TG }\end{array}$ & $\begin{array}{l}\text { - Total cholesterol, LDL-C } \\
\text { and TG for CP group }\end{array}$ \\
\hline $\begin{array}{l}\text { Noack } \\
\quad \text { et al., } 2000\end{array}$ & $\begin{array}{l}56 \text { individuals with } \\
\text { IGT } \\
17 \text { individuals with } \\
\text { hyperlipidemia } \\
27 \mathrm{SH} \text { control } \\
\text { individuals }\end{array}$ & Germany & $40-70$ & $\begin{array}{l}\text { - } \mathrm{PD} \\
\text { - } \mathrm{AL} \\
\text { - } \mathrm{BOP} \\
\text { - } \mathrm{PI}\end{array}$ & $\begin{array}{l}\text { - Total cholesterol } \\
\text { - TG }\end{array}$ & $\begin{array}{l}\text { - }>\text { No. sextants with } \\
\text { increased PD in } \\
\text { hyperlipidemia group } \\
\text { compared with control } \\
\text { individuals } \\
\text { + correlation between } \\
\text { serum TG levels and PD in } \\
\text { the overall sample }\end{array}$ \\
\hline $\begin{array}{l}\text { Wu } \\
\text { et al., } 2000\end{array}$ & $\begin{array}{l}\text { 10,146 participants } \\
\text { from NHANES III }\end{array}$ & USA & $\begin{array}{l}40.37 \pm \\
17.28 \\
(\text { mean } \pm S D)\end{array}$ & $\begin{array}{l}\text { - No disease: no } \\
\text { tooth examined } \\
\text { with } P D \geq 2 \mathrm{~mm} \text { or } \\
\mathrm{AL} \geq 3 \mathrm{~mm} \\
\text { - } \text { Mild } C P, \geq 1 \\
\text { examined tooth } \\
\text { with } \mathrm{PD} \geq 2 \mathrm{~mm} \text { or } \\
\mathrm{AL} \geq 3 \\
\mathrm{CP} \geq 1 \text { tooth with } \\
\mathrm{PD} \geq 3 \mathrm{~mm} \text { or } \mathrm{AL} \\
\geq 4 \mathrm{~mm}\end{array}$ & $\begin{array}{l}\text { - Total cholesterol } \\
\text { - HDL-C }\end{array}$ & $\begin{array}{l}\text { There was a weak } \\
\text { association between } \\
\text { cholesterol level and } \\
\text { periodontal status } \\
\text { - There was no association } \\
\text { between HDL-C and } \\
\text { periodontal status }\end{array}$ \\
\hline $\begin{array}{l}\text { Katz et al., } \\
2001\end{array}$ & $\begin{array}{l}151 \text { individuals } \\
\text { diagnosed as } \\
\text { having } \mathrm{CHD}, \mathrm{DM} \\
\text { or hypertension } \\
943 \mathrm{SH} \text { individuals }\end{array}$ & Israel & $26-53$ & - CPITN & $\begin{array}{l}\text { - Total cholesterol } \\
\text { - TG }\end{array}$ & $\begin{array}{l}\text { - Persons with } \\
\text { hypercholesterolemia had } \\
\text { more severe CP, } \\
\text { according to CPITN score } \\
\text { - There was no association } \\
\text { between TG and } \\
\text { periodontal status }\end{array}$ \\
\hline $\begin{array}{l}\text { Katz et al., } \\
2002\end{array}$ & $\begin{array}{l}9421 \text { military men } \\
1169 \text { military } \\
\text { women }\end{array}$ & Israel & $19-61$ & - CPITN & $\begin{array}{l}\text { - } \text { Total cholesterol } \\
\text { - } \mathrm{HDL}-\mathrm{C} \\
\text { - } \mathrm{LDL}-\mathrm{C} \\
\text { - } \mathrm{TG}\end{array}$ & $\begin{array}{l}\text { - A CPITN score of } 4 \text { is } \\
\text { strongly associated } \\
\text { with total and LDL-C } \\
\text { cholesterol and negatively } \\
\text { associated with HDL-C } \\
\text { in men }\end{array}$ \\
\hline $\begin{array}{l}\text { Craig et al., } \\
2003\end{array}$ & $\begin{array}{l}25 \mathrm{PH} \text { persons } \\
44 \mathrm{CP} \text { persons }\end{array}$ & USA & $\begin{array}{l}29.9 \pm 1.1 \\
38.7 \pm 1.3 \\
\text { respectively } \\
(\text { mean } \pm \text { SD) }\end{array}$ & $\begin{array}{l}\text { - } \geq 20 \text { teeth and } \geq \\
4 \text { sites with } P D>3 \\
\mathrm{~mm} \text { and } \geq 4 \text { sites } \\
\text { with } \mathrm{AL}>3 \mathrm{~mm}\end{array}$ & $\begin{array}{ll}\text { - } & \text { Total } \\
& \text { cholesterol } \\
\text { - } & \text { HDL-C } \\
\text { - } & \text { LDL-C } \\
\text { - } & \text { TG }\end{array}$ & $\begin{array}{l}\text { - } \quad \text { Total cholesterol and } \\
\text { LDL-C levels for CP group } \\
\text { - } \quad \text { < HDL-C for CP group } \\
\text { - } \quad>\text { TG levels for CP } \\
\text { persons, but without a } \\
\text { statistical significance }\end{array}$ \\
\hline
\end{tabular}


Table 5. (continued)

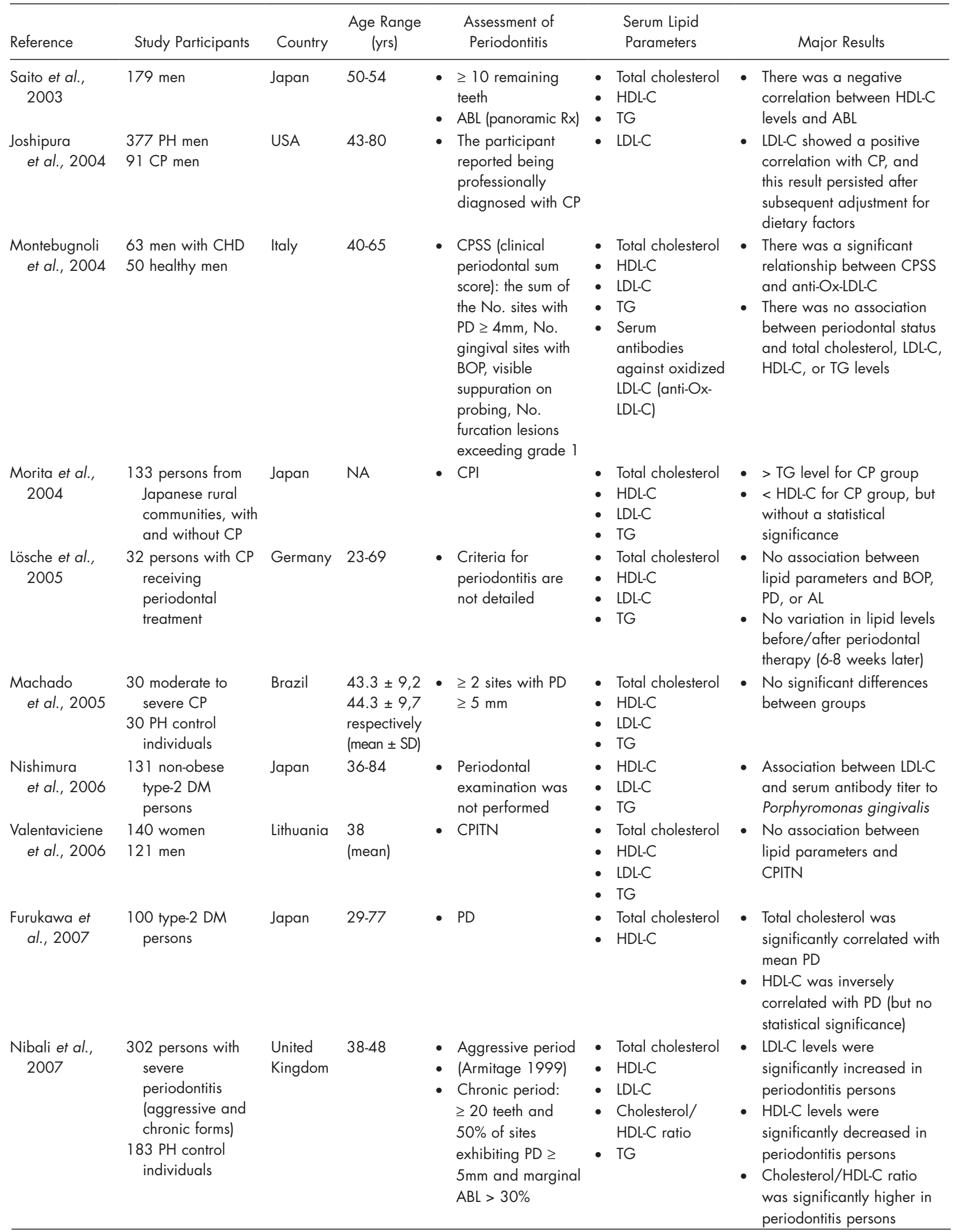


Table 5. (continued)

\begin{tabular}{|c|c|c|c|c|c|c|}
\hline Reference & Study Participants & Country & $\begin{array}{c}\text { Age Range } \\
\text { (yrs) }\end{array}$ & $\begin{array}{l}\text { Assessment of } \\
\text { Periodontitis }\end{array}$ & $\begin{array}{l}\text { Serum Lipid } \\
\text { Parameters }\end{array}$ & Major Results \\
\hline $\begin{array}{l}\text { Oz et al., } \\
2007\end{array}$ & $\begin{array}{l}51 \text { periodontitis } \\
\text { persons assigned } \\
\text { to the treatment } \\
\text { or control groups }\end{array}$ & Turkey & $36-66$ & $\begin{array}{l}->3 \text { sites with } P D \geq \\
\quad 4 \mathrm{~mm}\end{array}$ & $\begin{array}{l}\text { - } \text { Total cholesterol } \\
\text { - } \text { HDL-C } \\
\text { - } \quad \text { LDL-C } \\
\text { - } \quad \mathrm{TG}\end{array}$ & $\begin{array}{l}\text { - There was a significant } \\
\text { decrease in cholesterol } \\
\text { and LDL-C levels after } \\
\text { treatment ( } 3 \text { months later) }\end{array}$ \\
\hline $\begin{array}{l}\text { Türkoğlu et al., } \\
\quad 2008\end{array}$ & $\begin{array}{l}72 \text { persons, divided } \\
\text { into healthy } \\
\text { control } \\
\text { individuals, } \\
\text { PH-EHT, G-EHT, } \\
\text { CP-EHT }\end{array}$ & Turkey & $\begin{array}{l}44.83 \pm \\
8.4454 .35 \pm \\
11.14 \\
49.71 \pm 7.30 \\
54.38 \pm 6.76 \\
\text { respectively } \\
\text { (mean } \pm \text { SD) }\end{array}$ & $\begin{array}{l}\text { - } \geq 4 \text { non-adjacent } \\
\text { teeth with sites } \\
\text { with } \mathrm{AL} \geq 4 \mathrm{~mm} \\
\text { and } \mathrm{PD} \geq 5 \mathrm{~mm}, \\
\text { BOP at }>50 \% \text { of } \\
\text { sites }\end{array}$ & - Oxidized LDL & $\begin{array}{l}\text { No differences in } \\
\text { oxidized-LDL-C between } \\
\text { groups }\end{array}$ \\
\hline
\end{tabular}

* $\mathrm{ABL}$, alveolar bone loss; $\mathrm{AL}$, attachment loss; $\mathrm{BOP}$, bleeding on probing; CHD, coronary heart disease; CP, periodontitis; CPITN, community periodontal index of treatment needs; DM, diabetes mellitus; EHT, essential hypertension; G, gingivitis; HDL-C, high-density lipoprotein-cholesterol; LDL-C, low-density lipoprotein-cholesterol; NA, not available; NHANES III, Third National Health and Nutrition Examination Survey; PD, probing depth; $\mathrm{PH}$, periodontally healthy; PI, plaque index; SD, standard deviation; SH, systemically healthy.

Table 6. Published Reports about Arterial Hypertension and Periodontitis in Humans

\begin{tabular}{|c|c|c|c|c|c|c|}
\hline Reference & Study Participants & Country & $\begin{array}{l}\text { Mean Age } \\
(y r s \pm S D)\end{array}$ & $\begin{array}{l}\text { Assessment of } \\
\text { Periodontitis }\end{array}$ & $\begin{array}{l}\text { Vascular } \\
\text { Parameters }\end{array}$ & Major Results \\
\hline $\begin{array}{l}\text { Al-Emadi } \\
\quad \text { et al., } 2006\end{array}$ & $\begin{array}{l}210 \text { moderate or } \\
\text { severe } \mathrm{CP} \\
210 \mathrm{PH} \text { or mild } \mathrm{CP}\end{array}$ & USA & $\begin{array}{l}46.95 \\
( \pm 16.17)\end{array}$ & $\begin{array}{l}\text { - } \geq 20 \text { remaining } \\
\text { teeth } \\
\text { - } \text { Moderate/severe } \\
\text { CP: mean } A B L^{*} \\
\geq 2.5 \mathrm{~mm}\end{array}$ & - Self-reported HBP & $\begin{array}{l}\text { - }>\text { HBP prevalence in } \\
\text { subjetcs with moderate } \\
\text { to severe ABL (34.3\% } \\
\text { vs. } 7.6 \%)\end{array}$ \\
\hline $\begin{array}{l}\text { Engström } \\
\quad \text { et al., } 2007\end{array}$ & $\begin{array}{l}54 \text { persons with } \\
\text { known HBP } \\
141 \text { persons with } \\
\text { diastolic BP > } 90 \\
\text { mmHg during study } \\
195 \text { control persons }\end{array}$ & Sweden & $\begin{array}{l}\text { Mean age in } \\
\text { case groups: } \\
49 \text { and } 54, \\
\text { respectively }\end{array}$ & $\begin{array}{l}\text { - No. sites } \geq 5 \\
\text { (excluding the third } \\
\text { molar) }\end{array}$ & $\begin{array}{l}\text { - Diastolic and } \\
\text { systolic blood } \\
\text { pressure }\end{array}$ & $\begin{array}{l}\text { - }>\text { No. sites } \geq 5 \mathrm{~mm} \text { in } \\
\text { HBP persons } \\
\text { - Association between } \\
\text { prevalence of deep } \\
\text { periodontal pockets } \\
\text { and BP status }\end{array}$ \\
\hline $\begin{array}{l}\text { Higashi } \\
\quad \text { et al., } 2008\end{array}$ & $\begin{array}{l}32 \mathrm{CP}+, \mathrm{HBP}- \\
20 \text { control } \\
\text { individuals } \\
38 \mathrm{CP}+, \mathrm{HBP}+ \\
24 \mathrm{CP}_{-}, \mathrm{HBP}+\end{array}$ & Japan & $\begin{array}{l}25 \pm 3 \\
26 \pm 3 \\
\text { respectively } \\
54 \pm 13,56 \\
\pm 12 \\
\text { respectively }\end{array}$ & $\begin{array}{l}\text { - Self-reported } \\
\text { periodontal status }\end{array}$ & $\begin{array}{l}\text { - Forearm blood } \\
\text { flow (FBF) } \\
\text { response to } \\
\text { acetylcholine } \\
\text { (ACh) }\end{array}$ & $\begin{array}{l}\text { - }<\text { FBF response to } \mathrm{ACh} \\
\text { in both } \mathrm{CP} \text { groups } \\
\text { - Periodontal therapy } \uparrow \\
\text { ACh-induced } \\
\text { vasodilation in } \mathrm{CP} \\
\text { individuals }\end{array}$ \\
\hline $\begin{array}{l}\text { Holmlund } \\
\text { et al., } 2006\end{array}$ & $\begin{array}{l}\text { 3,352 persons with } \\
\text { history of HBP or } \\
\text { myocardial } \\
\text { infarction } \\
902 \text { control } \\
\text { individuals }\end{array}$ & Sweden & $45 \pm 17$ & $\begin{array}{l}\text { Periodontal disease } \\
\text { severity index } \\
\text { (PDSI), combining } \\
\text { ABL and BOP }\end{array}$ & $\begin{array}{l}\text { HBP was defined } \\
\text { as drug treatment } \\
\text { for this disease }\end{array}$ & $\begin{array}{l}\text { - Association between } \\
\text { severity of } \mathrm{CP} \text { and No. } \\
\text { of periodontal pockets } \\
\text { with HBP }\end{array}$ \\
\hline
\end{tabular}


Table 6. (continued)

\begin{tabular}{|c|c|c|c|c|c|c|}
\hline Reference & Study Participants & Country & $\begin{array}{l}\text { Mean Age } \\
\text { (yrs } \pm \text { SD) }\end{array}$ & $\begin{array}{l}\text { Assessment of } \\
\text { Periodontitis }\end{array}$ & $\begin{array}{l}\text { Vascular } \\
\text { Parameters }\end{array}$ & Major Results \\
\hline $\begin{array}{l}\text { Khader et al... } \\
2003\end{array}$ & $\begin{array}{l}603 \text { participants } \\
\text { from general } \\
\text { population }\end{array}$ & Jordan & 15-65 (range) & $\begin{array}{l}\text { - } \mathrm{PD}, \mathrm{AL}, \mathrm{PI} \\
\text { - } \text { Gingival recession }\end{array}$ & $\begin{array}{l}\text { - Questionnaire for } \\
\text { general health }\end{array}$ & $\begin{array}{l}\text { - Having HBP was } \\
\text { significantly associated } \\
\text { with increased AL and } \\
\text { gingival recession }\end{array}$ \\
\hline $\begin{array}{l}\text { Türkoğlu et al., } \\
2008\end{array}$ & $\begin{array}{l}72 \text { individuals, } \\
\text { divided into } \\
\text { healthy control } \\
\text { individuals, } \\
\text { PH-EHT, G-EHT, } \\
\text { CP-EHT }\end{array}$ & Turkey & $\begin{array}{l}44.83 \pm 8.44 \\
54.35 \pm \\
11.14,49.71 \\
\pm 7.30,54.38 \\
\pm 6.76 \\
\text { respectively }\end{array}$ & $\begin{array}{l}\text { - } \geq 4 \text { non-adjacent } \\
\text { teeth with sites with } \\
\mathrm{AL} \geq 4 \mathrm{~mm} \text { and } \mathrm{PD} \geq \\
5 \mathrm{~mm}, \mathrm{BOP} \text { at } \\
>50 \% \text { of sites }\end{array}$ & $\begin{array}{l}\text { - Serum } \lg M \\
\text { and } \lg G \\
\text { anti-cardiolipin }\end{array}$ & $\begin{array}{l}\text { - }>\text { mean } \lg M \text { anti-CL } \\
\text { levels and > } \\
\text { prevalence of persons } \\
\text { with IgM anti-CL+ in } \\
\text { CP-EHT group }\end{array}$ \\
\hline $\begin{array}{l}\text { Wakai et al., } \\
1999\end{array}$ & $\begin{array}{l}517 \text { males } \\
113 \text { females }\end{array}$ & Japan & $23-83$ & - CPITN & - Blood pressure & $\begin{array}{l}->\text { systolic BP was } \\
\text { associated with > CPITN }\end{array}$ \\
\hline
\end{tabular}

* $\mathrm{ABL}$, alveolar bone lose; $\mathrm{AL}$, clinical attachment level; anti-CL, anticardiolipin antibodies; $\mathrm{BOP}$, bleeding on probing; $\mathrm{BP}$; blood pressure; $\mathrm{CP}$, periodontitis; $\mathrm{CPI}$, community periodontal index; CPITN, community periodontal index of treatment needs; DM, diabetes mellitus; EHT, essential hypertension; FBF, forearm blood flow; G, gingivitis; HBP, hypertension; LDL-C, low-density lipoprotein-cholesterol; LVM, left ventricular mass; $\mathrm{OHI}$, oral hygiene index; $\mathrm{PD}$, probing depth; $\mathrm{PH}$, periodontally healthy; $\mathrm{Pl}$, plaque index.

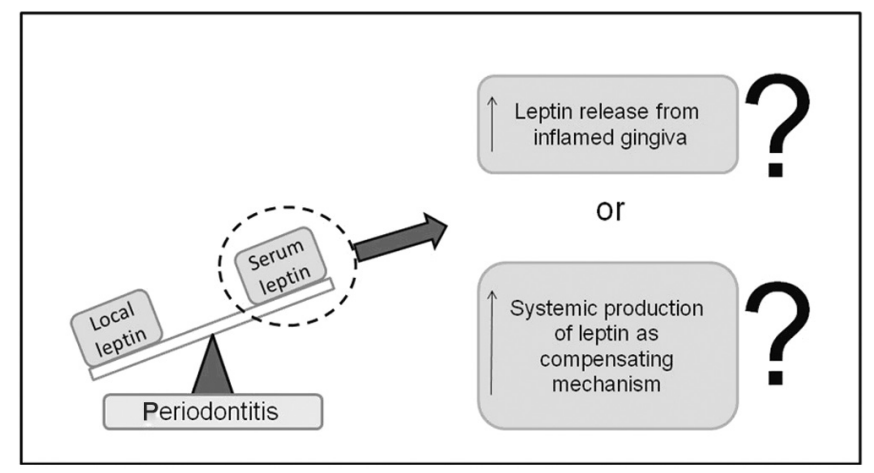

Figure 1. Increased serum leptin levels in periodontitis. Two putative explanations have been proposed. First, gingival inflammation would result in vasodilation, which might increase the net rate of leptin removal from the gingiva and increase serum leptin levels. The second hypothesis states that serum leptin levels rise as a body defense mechanism to counteract periodontal inflammation (according to Karthikeyan and Pradeep, 2007b).

obesity, insulin resistance, and diabetes, and in cardiovascular disease with increasing severity (Matsuzawa et al., 2004). This reduction appears to precede these disorders, and low adiponectin levels have been shown to predict the development of insulin resistance and type 2 diabetes (Spranger et al., 2003; Yamamoto et al., 2004). Thus, adiponectin has a key role in the development of MetS.

Currently, there is no report of adiponectin in periodontal tissues or GCF. By an in vitro approach, adiponectin acts as a potent negative regulator of the osteoclast formation induced by Aggregatibacter actinomycetemcomitans lipopolysaccharide (LPS). Therefore, adiponectin could exert an anti-inflammatory effect in periodontitis sites, and thereby have a negative influence over the onset and progression of periodontitis (Yamaguchi et al., 2007). In humans, two recent studies (Furugen et al., 2008; Saito et al., 2008) have found that serum adiponectin levels tended to decrease in Japanese persons with periodontitis, albeit not significantly. Moreover, adiponectin levels were negatively correlated with mean attachment loss, but not mean probing depth or percentage of sites bleeding on probing. Another study (Iwamoto et al., 2003) found that serum adiponectin levels did not change significantly after periodontal therapy. However, small sample size, lack of a control group, and a longer period for additional measures are relevant limitations in this study.

\section{Resistin and Periodontitis}

In humans, resistin is mainly secreted by monocytes, macrophages, and bone marrow, but also by adipocytes. It has a potent pro-inflammatory action. This molecule has been associated with insulin resistance in mice (Koerner et al., 2005). Two recent studies confirm the role of this molecule in periodontitis; serum resistin levels are higher in persons with periodontitis than in control individuals, and there is a positive correlation with bleeding on probing (Furugen et al., 2008; Saito et al., 2008). 


\section{OXIDATIVE STRESS AS A POTENTIAL LINK BETWEEN PERIODONTITIS AND METABOLIC SYNDROME}

As previously mentioned, oxidative stress is one of the main factors studied to explain the pathophysiological mechanisms of inflammatory conditions, such as MetS and periodontitis. A recent and exhaustive review (Chapple and Matthews, 2007) has described the complex role of oxidative stress in relation to periodontal breakdown. It seems that peripheral blood neutrophil hyperactivity in chronic and aggressive periodontitis exists as a constitutional element (Matthews et al., 2007a,b), rather than being entirely the result of peripheral priming by cytokines or plaque bacterial LPS. In addition, there may be possible baseline hyperactivity, with low-level extracellular ROS release in the absence of any exogenous stimulus in persons with periodontitis (Gustafsson and Asman, 1996; Gustafsson et al., 1997; Fredriksson et al., 1998; Matthews et al., 2007b).

There is increasing evidence for compromised anti-oxidant capacity in periodontal tissues and fluids, independent of smoking, and increased AGE levels in persons with type 2 diabetes and in smokers, which are risk factors for periodontitis. Such oxidation products can increase neutrophil adhesion, chemotaxis, and priming in hyper-reactive neutrophils, and might augment the damaging effects of the resultant oxidative stress (Brock et al., 2004; Palmer et al., 2005; Panjamurthy et al., 2005). In addition to this, the up-regulation of pro-inflammatory transcription factors, such as NF- $\mathrm{KB}$ and activating protein-1, in inflamed periodontal tissues contributes to reduced glutathione depletion and ROS generation (Chapple, 1997; JanssenHeininger et al., 2000).

It is important to emphasize the influence of periodontitis on serum and/or plasma oxidative markers in humans. Several studies have demonstrated an increase in products of oxidative damage in peripheral blood from persons with periodontitis compared with control individuals (Battino et al., 2001; Montebugnoli et al., 2004; Baltacioglu et al., 2008). Moreover, it is evidence of a decreased anti-oxidant capacity in persons with periodontitis, evaluated by different assays (Chapple et al., 2002, 2007; Battino et al., 2003; Brock et al., 2004; Panjamurthy et al., 2005; Baltacioglu et al., 2006; Akalin et al., 2007; Konopka et al., 2007; Zilinskas et al., 2007).

Therefore, it might be argued that this increased pro-oxidative state and decreased anti-oxidant capacity in persons with periodontitis could facilitate the onset of a decrease in insulin sensitivity, which could be aggravated by a high fat diet in these persons. This is in agreement with the previously mentioned report which demonstrated that an increase in ROS production precedes insulin resistance (Matsuzawa-Nagata et al., 2008). Conversely, the presence of MetS or any of its components in a previously periodontally healthy person could facilitate a prooxidant state which would diminish anti-oxidant capacity of the periodontal tissues, and the response of these tissues to bacterial challenge could be impaired. The presence of a high RAGE expression in periodontal tissues (Katz et al., 2005) is an important finding supporting the sensitivity of these tissues to products derived from oxidative damage. Moreover, AGE may

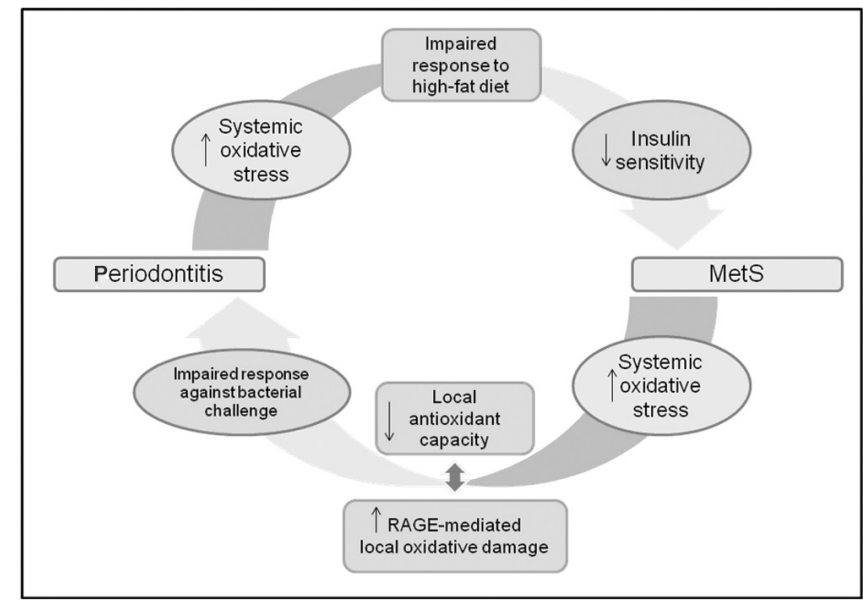

Figure 2. Hypothetic role of oxidative stress as a link between periodontitis and metabolic syndrome (MetS).

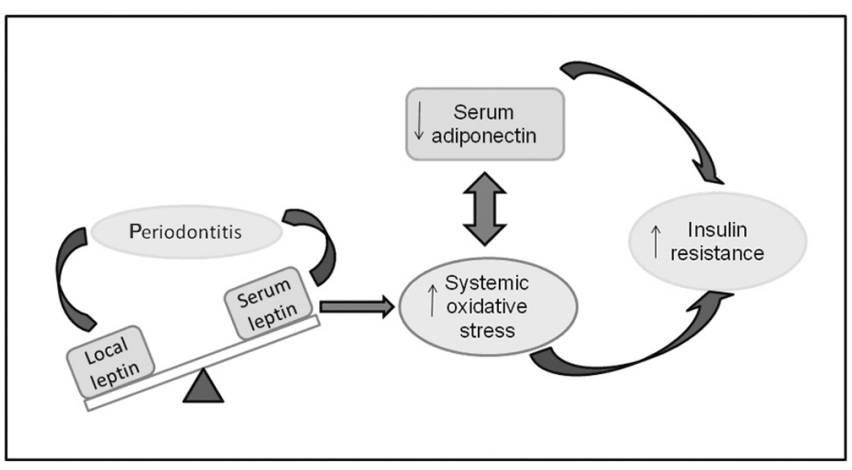

Figure 3. Proposed roles of leptin and adiponectin as putative mediators in the oxidative imbalance enhancing periodontitis and insulin resistance.

promote apoptosis in osteoblasts (Alikhani et al., 2007a) and fibroblasts (Alikhani et al., 2007b), and this might have an influence on alveolar bone homeostasis and the progression of periodontitis. This way, it is plausible to draw a patho-physiological picture in which a bidirectional influence exists between both conditions, with oxidative stress as a common link (Fig. 2).

Leptin and adiponectin appear as potential candidates to influence in a positive or negative manner, respectively, over the pro-oxidative state in periodontitis and MetS. Leptin has been demonstrated to have a predominantly pro-oxidative effect (Bouloumie et al., 1999; Maingrette and Renier, 2003; Suzuki et al., 2003; Beltowski et al., 2004). The increased serum leptin levels existing in persons with periodontitis, possibly in an attempt to modulate the immune response, could be one of the factors that induces oxidative stress and accelerates the onset of insulin resistance. This increased oxidative stress could facilitate a relative hypoadiponectinemia (Hattori et al., 2005; Soares et al., 2005; Katsuki et al., 2006), like that presenting in persons with periodontitis, which could also decrease the protection against oxidative damage, since this adipocytokine has been demonstrated to have a protective effect against oxidative stress (Nakanishi et al., 2005; Barazzoni et al., 2007). This picture is ideal for the onset of features comprising MetS (Fig. 3). 


\section{CONCLUSIONS}

The evidence reviewed indicates that there is a need for further research concerning the possible relationships between MetS and periodontitis, in relation to both local and systemic health and disease. But according to the available reviewed data, we can propose some pathological mechanisms that could explain this relationship. Adipocytokines may act as a link between both conditions, and relevant questions arise which could guide future research: (1) Can gingival keratinocytes or any cell type from gingiva or periodontal ligament express leptin, adiponectin, and/ or resistin, or any of their respective receptors? (2) How do these molecules interact with other cytokines in periodontal tissues and influence oxidative stress derived from periodontal breakdown? (3) What is the relationship between serum and GCF levels of these molecules in systemically healthy and MetS persons, with or without chronic gingivitis and/or periodontitis?

Oxidative stress could be a common mechanism in the development of several features related to both MetS and periodontitis, and perhaps an interaction between both conditions may result in a worse evolution of both of them. To elucidate the potential association between both conditions, several approaches are suggested:

(1) Design large-scale studies to assess biomarkers of oxidative stress and anti-oxidant defenses in persons suffering from MetS, with and without periodontitis at several grades of severity, to facilitate comparisons between both conditions and to determine whether periodontitis can affect redox state in persons with MetS. Use of the new definition of this condition (IDF, 2005), applied to different ethnic groups, would be an important element, with the aim of achieving more reliable conclusions.

(2) Analysis of biomarkers of oxidative stress, to obtain a more complete view of the potential interactions between both conditions. All such markers should be analyzed in relation to each component of MetS in persons with and without periodontitis.

(3) Design an intervention study, in which the influence of periodontal therapy (conservative and surgery) on biomarkers of oxidative stress in persons with MetS would be assessed.

All data derived from these and other such approaches would increase the knowledge of the possible interrelationships among periodontitis, MetS, and CVD, with the natural aim of improving their diagnosis, treatment, and, ultimately, prevention.

\section{ACKNOWLEDGMENTS}

Funding for this research was from Università Politecnica delle Marche, Italy, and Grupo de investigación CTS113 Junta de Andalucia, Spain.

\section{REFERENCES}

Akalin FA, Baltacioglu E, Alver A, Karabulut E (2007). Lipid peroxidation levels and total oxidant status in serum, saliva and gingival crevicular fluid in patients with chronic periodontitis. $J$ Clin Periodontol 34:558-565.
Akalin FA, Isiksal E, Baltacioglu E, Renda N, Karabulut E (2008). Superoxide dismutase activity in gingiva in type-2 diabetes mellitus patients with chronic periodontitis. Arch Oral Biol 53:44-52.

Al-Emadi A, Bissada N, Farah C, Siegel B, Al-Zaharani M (2006). Systemic diseases among patients with and without alveolar bone loss. Quintessence Int 37:761-765.

Alberti KG, Zimmet PZ (1998). Definition, diagnosis and classification of diabetes mellitus and its complications. Part 1: diagnosis and classification of diabetes mellitus provisional report of a WHO consultation. Diabet Med 15:539-553.

Alikhani M, Alikhani Z, Boyd C, MacLellan CM, Raptis M, Liu R, et al. (2007a). Advanced glycation endproducts stimulate osteoblast apoptosis via the MAP kinase and cytosolic apoptotic pathways. Bone 40:345-353

Alikhani M, Maclellan CM, Raptis M, Vora S, Trackman PC, Graves DT (2007b). Advanced glycation end products induce apoptosis in fibroblasts through activation of ROS, MAP kinases, and the FOXO1 transcription factor. Am J Physiol Cell Physiol 292:C850-C856.

American Diabetes Association (2005). Diagnosis and classification of diabetes mellitus. Diabetes Care 28 (Suppl 1):37-42.

Angeli F, Verdecchia P, Pellegrino C, Pellegrino RG, Pellegrino G, Prosciutti $\mathrm{L}$, et al. (2003). Association between periodontal disease and left ventricle mass in essential hypertension. Hypertension 41:488-492.

Armitage GC (1999). Development of a classification system for periodontal diseases and conditions. Ann Periodontol 4:1-6.

Balkau B, Charles MA, Drivsholm T, Borch-Johnsen K, Wareham N, Yudkin JS, et al. (2002). The European Group for the Study of Insulin Resistance (EGIR). Frequency of the WHO metabolic syndrome in European cohorts, and an alternative definition of an insulin resistance syndrome. Diabetes Med 28:364-376.

Baltacioglu E, Akalin FA, Alver A, Balaban F, Unsal M, Karabulut E (2006). Total antioxidant capacity and superoxide dismutase activity levels in serum and gingival crevicular fluid in post-menopausal women with chronic periodontitis. J Clin Periodontol 33:385-392.

Baltacioglu E, Akalin FA, Alver A, Deger O, Karabulut E (2008). Protein carbonyl levels in serum and gingival crevicular fluid in patients with chronic periodontitis. Arch Oral Biol 53:716-722.

Barazzoni R, Bernardi A, Biasia F, Semolic A, Bosutti A, Mucci M, et al. (2007). Low fat adiponectin expression is associated with oxidative stress in nondiabetic humans with chronic kidney disease: impact on plasma adiponectin concentration. Am J Physiol Regul Integr Comp Physiol 293:R47-R54.

Basta G, Schmidt AM, DeCaterina R (2004). Advanced glycation end products and vascular inflammation: implications for accelerated atherosclerosis in diabetes. Cardiovasc Res 63:582-592.

Battino M, Bullon P, Wilson M, Newman H (1999). Oxidative injury and inflammatory periodontal diseases: the challenge of anti-oxidants to free radicals and reactive oxygen species. Crit Rev Oral Biol Med 10:458-476

Battino M, Ferreiro MS, Bompadre S, Leone L, Mosca F, Bullon P (2001). Elevated hydroperoxide levels and antioxidant patterns in PapillonLefèvre syndrome. J Periodontol 72:1760-1766.

Battino M, Ferreiro MS, Quiles JL, Bompadre S, Leone L, Bullon P (2003). Alterations in the oxidation products, antioxidant markers, antioxidant capacity and lipid patterns in plasma of patients affected by PapillonLefèvre syndrome. Free Radic Res 37:603-609.

Baynes JW, Thorpe SR (2000). Glycoxidation and lipoxidation in atherogenesis. Free Radic Biol Med 28:1708-1716.

Beltowski J, Wójcicka G, Marciniak A, Jamroz A (2004). Oxidative stress, nitric oxide production, and renal sodium handling in leptin-induced hypertension. Life Sci 74:2987-3000.

Borges PK, Gimeno SG, Tomita NE, Ferreira SR (2007). Prevalence and characteristics associated with metabolic syndrome in JapaneseBrazilians with and without periodontal disease. Cad Saude Publica 23: 657-668.

Bouloumie A, Marumo T, Lafontan M, Busse R (1999). Leptin induces oxidative stress in human endothelial cells. FASEB $J$ 13:1231-1238.

Bozkurt FY, Yetkin Ay Z, Sütçü R, Delibaş N, Demirel R (2006). Gingival crevicular fluid leptin levels in periodontitis patients with long-term and heavy smoking. $J$ Periodontol 77:634-640. 
Brock GR, Matthews JB, Butterworth CJ, Chapple ILC (2004). Local and systemic antioxidant capacity in periodontitis and health. $J$ Clin Periodontol 31:515-521.

Bruce CR, Carey AL, Hawley JA, Febbraio MA (2003). Intramuscular heat shock protein 72 and heme oxygenase-1 mRNA are reduced in patients with type 2 diabetes: evidence that insulin resistance is associated with a disturbed antioxidant defence mechanism. Diabetes 52:2338-2345.

Cameron AJ, Shaw JE, Zimmet PZ (2004). The metabolic syndrome: prevalence in worldwide populations. Endocrinol Metab Clin North Am 33:351-375.

Ceriello A (2000). Oxidative stress and glycemic regulation. Metabolism 49:27-29.

Ceriello A, Motz E (2004). Is oxidative stress the pathogenic mechanism underlying insulin resístance, diabetes and cardiovascular disease? The common soil hypothesis revisited. Arterioscler Thromb Vasc Biol 24:816-823.

Chapple IL (1997). Reactive oxygen species and antioxidants in inflammatory diseases. J Clin Periodontol 24:287-296.

Chapple IL, Matthews JB (2007). The role of reactive oxygen and antioxidant species in periodontal tissue destruction. Periodontol 2000 43:160-232.

Chapple IL, Brock G, Eftimiadi C, Matthews JB (2002). Glutathione in gingival crevicular fluid and its relation to local antioxidant capacity in periodontal health and disease. Mol Pathol 55:367-373.

Chapple IL, Milward MR, Dietrich T (2007). The prevalence of inflammatory periodontitis is negatively associated with serum antioxidant concentrations. J Nutr 137:657-664.

Christgau M, Palitzsch KD, Schmalz G, Kreiner U, Frenzel S (1998). Healing response to non-surgical periodontal therapy in patients with diabetes mellitus: clinical, microbiological, and immunologic results. $J$ Clin Periodontol 25:112-124.

Collin HL, Sorsa T, Meurman JH, Niskanen L, Salo T, Rönkä H, et al. (2000). Salivary matrix metalloproteinase (MMP-8) levels and gelatinase (MMP-9) activities in patients with type 2 diabetes mellitus. $J$ Periodontal Res 35:259-265.

Cosentino F, Hishikawa K, Katusic ZS, Luscher TF (1997). High glucose increases nitric oxide synthase expression and superoxide anion generation in human aortic endothelial cells. Circulation 96:25-28.

Craig RG, Yip JK, So MK, Boylan RJ, Socransky SS, Haffajee AD (2003). Relationship of destructive periodontal disease to the acute-phase response. J Periodontol 74:1007-1016.

Cutler CW, Machen RL, Jotwani R, Iacopino AM (1999). Heightened gingival inflammation and attachment loss in type 2 diabetics with hyperlipidemia. $J$ Periodontol 70:1313-1321.

D'Aiuto F, Sabbah W, Netuveli G, Donos N, Hingorani AD, Deanfield J, et al. (2008). Association of the metabolic syndrome with severe periodontitis in a large U.S. population-based survey. J Clin Endocrin Metab 93:3989-3994.

Dandona P, Aljada A, Mohanty P (2002). The anti-inflammatory and potential anti-atherogenic effect of insulin: a new paradigm. Diabetologia 45:924-930.

Dandona P, Aljada A, Bandyopadhyay A (2004). Inflammation: the link between insulin resistance, obesity and diabetes. Trends Immunol 25:4-7.

De Toni S, Piva E, Lapolla A, Fontana G, Fedele D, Plebani M (1997). Respiratory burst of neutrophils in diabetic patients with periodontal disease. Ann NY Acad Sci 832:363-367.

Di Filippo C, Verza M, Coppola L, Rossi F, D’Amico M, Marfella R (2007). Insulin resistance and postprandial hyperglycemia the bad companions in natural history of diabetes: effects on health of vascular tree. Curr Diabetes Rev 3:268-273.

Eckel RH, Grundy SM, Zimmet PZ (2005). The metabolic syndrome. Lancet 365:1415-1428.

El-Haschimi K, Pierroz DD, Hileman SM, Björbaek C, Flier JS (2000). Two defects contribute to hypothalamic leptin resistance in mice with dietinduced obesity. $J$ Clin Invest 105:1827-1832.

Engström S, Gahnberg L, Högberg H, Svärdsudd K (2007). Association between high blood pressure and deep periodontal pockets: a nested case-referent study. Ups J Med Sci 112:95-103.

Esterbauer H, Schaur RJ, Zollner H (1991). Chemistry and biochemistry of 4-hydroxynonenal, malonaldehyde and related aldehydes. Free Radic Biol Med 11:81-128.
Esterbauer H, Gebicki J, Puhl H, Jürgens G (1992). The role of lipid peroxidation and antioxidants in oxidative modification of LDL. Free Radic Biol Med 13:341-390.

Evans JL, Goldfine ID, Maddux BA, Grodsky GM (2003). Are oxidative stress activated signaling pathways mediators of insulin resistance and $\beta$-cell dysfunction? Diabetes 52:1-8.

Fardi A, Papadimitriou D (2007). Periodontal and atherosclerosis-induced diseases. Int Angiol 26:197-205.

Fontana G, Lapolla A, Sanzari M, Piva E, Mussap M, De Toni S, et al. (1999). An immunological evaluation of type II diabetic patients with periodontal disease. J Diabetes Complications 13:23-30.

Ford PJ, Yamazaki K, Seymour GJ (2007). Cardiovascular and oral disease interactions: what is the evidence? Prim Dent Care 14:59-66.

Fredriksson M, Gustafsson A, Bergström K, Asman B (1998). Hyperreactive peripheral neutrophils in adult periodontitis: generation of chemiluminescence and intracellular hydrogen peroxide after in vitro priming and FcgammaR-stimulation. J Clin Periodontol 25:395-398.

Furugen R, Hayashida H, Yamaguchi N, Yoshihara A, Ogawa H, Miyazaki $\mathrm{H}$, et al. (2008). The relationship between periodontal condition and serum levels of resistin and adiponectin in elderly Japanese. $J$ Clin Periodontol 43:556-562.

Furukawa T, Wakai K, Yamanouchi K, Oshida Y, Miyao M, Watanabe T, et al. (2007). Associations of periodontal damage and tooth loss with atherogenic factors among patients with type 2 diabetes mellitus. Intern Med 46:1359-1364.

Ghanim H, Aljada A, Hofmeyer D, Syed T, Mohanty P, Dandona P (2004). Circulating mononuclear cells in the obese are in a pro-inflammatory state. Circulation 110:1564-1571.

Gołebiewska M, Taraszkiewicz-Sulik K, Kuklińska A, Musiał WJ (2006). Periodontal condition in patients with cardiovascular diseases. Adv Med Sci 51(Suppl 1):69-72.

Gonçalves D, Correa FO, Khalil NM, de Faria Oliveira OM, Orrico SR (2008). The effect of non-surgical periodontal therapy on peroxidase activity in diabetic patients: a case-control pilot study. JClin Periodontol 35:799-806.

Graves DT, Liu R, Oates TW (2007). Diabetes-enhanced inflammation and apoptosis: impact on periodontal pathosis. Periodontol 2000 45:128-137.

Grossi SG, Skrepcinski FB, DeCaro T, Robertson DC, Ho AW, Dunford RG, et al. (1997). Treatment of periodontal disease in diabetics reduces glycated hemoglobin. $J$ Periodontol 68:713-719.

Grundy SM (2006). Does a diagnosis of metabolic syndrome have value in clinical practice? Am J Clin Nutr 83:1248-1251.

Grundy SM, Brewer HB Jr, Cleeman JI, Smith SC Jr, Lenfant D (2004). Definition of metabolic syndrome: report of the National, Heart, Lung and Blood Institute/American Heart Association conference on scientific issues related to definition. Circulation 109:433-438.

Gustafsson A, Asman B (1996). Increased release of free oxygen radicals from peripheral neutrophils in adult periodontitis after Fcgammareceptor stimulation. J Clin Periodontol 23:38-44.

Gustafsson A, Asman B, Bergström K (1997). Priming response to inflammatory mediators in hyperreactive peripheral neutrophils from adult periodontitis. Oral Dis 3:167-171.

Guzik TJ, Mangalat D, Korbut R (2006). Adipocytokines-novel link between inflammation and vascular function? J Physiol Pharmacol 57:505-528.

Halliwell B (1991). Reactive oxygen species in living systems: source, biochemistry, and role in human disease. Am J Med 91:14-22.

Hansel B, Giral P, Nobecourt E, Chantepie S, Bruckert E, Chapman MJ, et al. (2004). Metabolic syndrome is associated with elevated oxidative stress and dysfunctional dense high-density lipoprotein particles displaying impaired antioxidative activity. J Clin Endocrinol Metab 89:4963-4971.

Hattori S, Hattori Y, Kasai K (2005). Hypoadiponectinemia is caused by chronic blockade of nitric oxide synthesis in rats. Metabolism 54:482-487.

Herring ME, Shah SK (2006). Periodontal disease and control of diabetes mellitus. J Am Osteopath Assoc 106:416-421.

Higashi Y, Goto C, Jitsuiki D, Umemura T, Nishioka K, Hidaka T, et al. (2008). Periodontal infection is associated with endothelial dysfunction in healthy subjects and hypertensive patients. Hypertension $51: 446-453$. 
Holmlund A, Holm G, Lind L (2006). Severity of periodontal disease and number of remaining teeth are related to the prevalence of myocardial infarction and hypertension in a study based on 4,254 subjects. J Periodontol 77:1173-1178.

Hotamisligil GS (2006). Inflammation and metabolic disorders. Nature 444:860-867.

IDF consensus worldwide definition of the metabolic syndrome (2005). Part 1. Worldwide definition for use in clinical practice. http://www.idf.org/ webdata/docs/idf_meta_def_final.pdf (accessed 2008-11-18).

Iwamoto Y, Nishimura F, Nakagawa M, Sugimoto H, Shikata K, Makino H, et al. (2001). The effect of antimicrobial periodontal treatment on circulating tumor necrosis factor-alpha and glycated hemoglobin level in patients with type 2 diabetes. $J$ Periodontol 72:774-778.

Iwamoto Y, Nishimura F, Soga Y, Takeuchi K, Kurihara M, Takashiba S, et al. (2003). Antimicrobial periodontal treatment decreases serum C-reactive protein, tumor necrosis factor-alpha, but not adiponectin levels in patients with chronic periodontitis. $J$ Periodontol 74:1231-1236.

Janssen-Heininger YMW, Poynter ME, Baeuerle PA(2000). Recent advances towards understanding redox mechanisms in the activation of nuclear factor кB. Free Radic Biol Med 28:1317-1327.

Jenkins AJ, Best JD, Klein RL, Lyons TJ (2004). Lipoproteins, glycoxidation and diabetic angiopathy. Diabetes Metab Res Rev 20:349-368

Johnson RB, Serio FG (2001). Leptin within healthy and diseased human gingiva. J Periodontol 72:1254-1257.

Joshipura KJ, Wand HC, Merchant AT, Rimm EB (2004). Periodontal disease and biomarkers related to cardiovascular disease. $J$ Dent Res 83:151-155.

Juge-Aubry CE, Henrichot E, Meier CA (2005). Adipose tissue: a regulator of inflammation. Best Pract Res Clin Endocrinol Metab 19:547-566.

Kahn R (2007). Metabolic syndrome: is it a syndrome? Does it matter? Circulation 115:1806-1811.

Kahn R (2008). Metabolic syndrome: what is the clinical usefulness? Lancet 371:1891-1893.

Kahn R, Buse J, Ferrandini E, Stern M (2005). The metabolic syndrome: time for a critical appraisal. Joint statement from the American Diabetes Association and the European Association for the Study of Diabetes. Diabetes Care 28:2289-2304.

Karthikeyan BV, Pradeep AR (2007a). Leptin levels in gingival crevicular fluid in periodontal health and disease. J Periodontal Res 42:300-304.

Karthikeyan BV, Pradeep AR (2007b). Gingival crevicular fluid and serum leptin: their relationship to periodontal health and disease. $J$ Clin Periodontol 34:467-472.

Katsuki A, Suematsu M, Gabazza EC, Murashima S, Nakatani K, Togashi K, et al. (2006). Increased oxidative stress is asociated with decreased circulating levels of adiponectin in Japanese metabolically obese, normal-weight men with normal glucose tolerance. Diabetes Res Clin Pract 73:310-314.

Katz J, Chaushu G, Sharabi Y (2001). On the association between hypercholesterolemia, cardiovascular disease and severe periodontal disease. J Clin Periodontol 28:865-868.

Katz J, Flugelman MY, Goldberg A, Heft M (2002). Association between periodontal pockets and elevated cholesterol and low density lipoprotein cholesterol levels. J Periodontol 73:494-500.

Katz J, Bhattacharyya I, Farkhondeh-Kish F, Perez FM, Caudle RM, Heft MW (2005). Expression of the receptor of advanced glycation end products in gingival tissues of type 2 diabetes patients with chronic periodontal disease: a study utilizing immunohistochemistry and RT-PCR. J Clin Periodontol 32:40-44.

Khader YS, Rice JC, Lefante JJ (2003). Factors associated with periodontal diseases in a dental teaching clinic population in northern Jordan. $J$ Periodontol 74:1610-1617.

Koerner A, Kratzsch J, Kiess W (2005). Adipocytokines: leptin-the classical, resistin-the controversial, adiponectin-the promising, and more to come. Best Pract Res Clin Endocrinol Metab 19:525-546.

Konopka T, Król K, Kopec W, Gerber H (2007). Total antioxidant status and 8-hydroxy-2'-deoxyguanosine levels in gingival and peripheral blood of periodontitis patients. Arch Immunol Ther Exp 55:417-422.

Koyama H, Shoji T, Yokoyama H, Motoyama K, Mori K, Fukumoto S, et al. (2005). Plasma level of endogenous secretory RAGE is associated with components of the metabolic syndrome and atherosclerosis. Arterioscler Thromb Vasc Biol 25:2587-2593.

Kuo LC, Polson AM, Kang T (2008). Associations between periodontal diseases and systemic diseases: a review of the inter-relationships and interactions with diabetes, respiratory diseases, cardiovascular diseases and osteoporosis. Public Health 122:417-433.

Leite CL, Redins CA, Vasquez EC, Meyrelles SS (2005). Experimentalinduced periodontitis is exacerbated in spontaneously hypertensive rats. Clin Exp Hypertens 27:523-531.

Liu R, Bal HS, Desta T, Krothapalli N, Alyassi M, Luan Q, et al. (2006). Diabetes enhances periodontal bone loss through enhanced resorption and diminished bone formation. J Dent Res 85:510-514.

Lösche W, Karapetow F, Pohl A, Pohl C, Kocher T (2000). Plasma lipid and blood glucose levels in patients with destructive periodontal disease. $J$ Clin Periodontol 27:537-541.

Lösche W, Marshal GJ, Apatzidou DA, Krause S, Kocher T, Kinane DF (2005). Lipoprotein-associated phospholipase $\mathrm{A}_{2}$ and plasma lipids in patients with destructive periodontal disease. J Clin Periodontol 32:640-644.

Machado AC, Quirino MR, Nascimento LF (2005). Relation between chronic periodontal disease and plasmatic levels of triglycerides, total cholesterol and fractions. Braz Oral Res 19:284-289.

Maddux BA, See W, Lawrence JC Jr, Goldfine AL, Goldfine ID, Evans JL (2001). Protection against oxidative stress-induced insulin resistance in rat L6 muscle cells by micromolar concentrations of $\alpha$-lipoic acid. Diabetes 50:404-410.

Maingrette F, Renier G (2003). Leptin increases lipoprotein lipase secretion by macrophages: involvement of oxidative stress and protein kinase $\mathrm{C}$. Diabetes 52:2121-2128.

Margetic S, Gazzola C, Pegg GG, Hill RA (2002). Leptin: a review of its peripheral actions and interactions. Int $J$ Obes Relat Metab Disord 26:1407-1433.

Matsuzawa Y, Funahashi T, Kihara S, Shimomura I (2004). Adiponectin and metabolic syndrome. Arterioscler Thromb Vasc Biol 24:29-33.

Matsuzawa-Nagata N, Takamura T, Ando H, Nakamura S, Kurita S, Misu H, et al. (2008). Increased oxidative stress precedes the onset of high-fat diet-induced insulin resistance and obesity. Metabolism 57:1071-1077

Matthews JB, Wright HJ, Ling-Mountford N, Cooper PR, Chapple ILC (2007a). Neutrophil hyper-responsiveness in periodontitis. J Dent Res $86: 718-722$

Matthews JB, Wright HJ, Roberts A, Cooper PR, Chapple ILC (2007b). Hyperactivity and reactivity of peripheral blood neutrophils in chronic periodontitis. Clin Exp Immunol 147:255-264.

Mealey BL, Ocampo GL (2007). Diabetes mellitus and periodontal disease. Periodontol 2000 44:127-153.

Montebugnoli L, Servidio D, Miaton RA, Prati C, Tricoci P, Melloni C (2004). Poor oral health is associated with coronary heart disease and elevated systemic inflammatory and haemostatic factors. J Clin Periodontol 31:25-29.

Morita M, Horiuchi M, Kinoshita Y, Yamamoto T, Watanabe T (2004). Relationship between blood triglyceride levels and periodontal status. Community Dent Health 21:32-36.

Nakanishi S, Yamane K, Kamei N, Nojima H, Okubo M, Kohno N (2005). A protective effect of adiponectin against oxidative stress in Japanese Americans: the association between adiponectin or leptin and urinary isoprostane. Metabolism 54:194-199.

Navarro-Sanchez AB, Faria-Almeida R, Bascones-Martinez A (2007). Effect of non-surgical periodontal therapy on clinical and immunological response and glycaemic control in type 2 diabetic patients with moderate periodontitis. $J$ Clin Periodontol 34:835-843.

NCEP (2001). Executive summary of the third report of the National Cholesterol Education Program Expert Panel on detection, evaluation, and treatment of high blood cholesterol in adults (Adult Treatment Panel III). J Am Med Assoc 285:2486-2497.

Newman HN (1974). Diet, attrition, plaque and dental disease. Br Dent $J$ 136:491-497.

Nibali L, D’Aiuto F, Griffiths G, Patel K, Suvan J, Tonetti MS (2007). Severe periodontitis is associated with systemic inflammation and a dysmetabolic status: a case-control study. J Clin Periodontol 34:931-937. 
Nichols GA, Hillier TA, Brown JB (2007). Progression from newly acquired impaired fasting glusose to type 2 diabetes. Diabetes Care 30:228-233.

Ninomiya JK, L'Italien G, Criqui MH, Whyte JL, Gamst A, Chen RS (2004). Association of the metabolic syndrome with history of myocardial infarction and stroke in the Third National Health and Nutrition Examination Survey. Circulation 109:42-46.

Nishimura F, Taniguchi A, Yamaguchi-Morimoto M, Soga Y, Iwamoto Y, Kokeguchi S, et al. (2006). Periodontal infection and dyslipidemia in type 2 diabetics: association with increased HMG-CoA reductase expression. Horm Metab Res 38:530-535.

Nishimura F, Iwamoto Y, Soga Y (2007). The periodontal host response with diabetes. Periodontol 2000 43:245-253.

Njoroge FG, Monnier VM (1989). The chemistry of the Maillard reaction under physiological conditions: a review. Prog Clin Biol Res 304:85-107.

Noack B, Jachmann I, Roscher S, Sieber L, Kopprasch S, Lück C, et al. (2000). Metabolic diseases and their possible link to risk indicators of periodontitis. J Periodontol 71:898-903.

Oz SG, Fentoglu O, Kilicarslan A, Guven GS, Tanrtover MD, Aykac Y, et al. (2007). Beneficial effects of periodontal treatment on metabolic control of hypercholesterolemia. South Med J 100:686-691.

Palmer RM, Wilson RF, Hasan AS, Scott DA (2005). Mechanisms of action of environmental factors-tobacco smoking. J Clin Periodontol 32(Suppl 6):180-195.

Panjamurthy K, Manoharan S, Ramachandran CR (2005). Lipid peroxidation and antioxidant status in patients with periodontitis. Cell Mol Biol Lett 10:255-264

Paolisso G, Giugliano D (1996). Oxidative stress and insulin action. Is there a relationship? Diabetologia 39:357-363.

Paquette DW, Brodala N, Nichols TC (2007). Cardiovascular disease, inflammation, and periodontal infection. Periodontol 2000 44:113-126.

Perlstein MI, Bissada NF (1977). Influence of obesity and hypertension on the severity of periodontitis in rats. Oral Surg Oral Med Oral Pathol 43:707-719.

Pischon N, Heng N, Bernimoulin JP, Kleber BM, Willich SN, Pischon T (2007). Obesity, inflammation and periodontal disease. J Dent Res 86:400-409.

Pischon N, Pischon T, Kröger J, Gülmez E, Kleber BM, Bernimoulin JP, et al. (2008). Association among rheumatoid arthritis, oral hygiene, and periodontitis. $J$ Periodontol 79:979-986.

Pischon T, Hu FB, Rexrode KM, Girman CJ, Manson JE, Rimm EB (2008). Inflammation, the metabolic syndrome, and risk of coronary heart disease in women and men. Atherosclerosis 197:392-399.

Preshaw PM, Foster N, Taylor JJ (2007). Cross-susceptibility between periodontal disease and type 2 diabetes mellitus: an immunobiological perspective. Periodontol 2000 45:138-157.

Reaven GM (1988). Banting lecture 1988. Role of insulin resistance in human disease. Diabetes 37:1595-1607.

Reaven GM (2005). The metabolic syndrome: requiescat in pace. Clin Chem 51:931-938

Reaven GM (2006). The metabolic syndrome: is this diagnosis necessary? Am J Clin Nutr 83:1237- 1247; erratum in Am J Clin Nutr 84:1253, 2006.

Rosen ED, Spiegelman BM (2006). Adipocytes as regulators of energy balance and glucose homeostasis. Nature 444:847-853.

Saito T, Shimazaki Y (2007). Metabolic disorders related to obesity and periodontal disease. Periodontol 2000 43:254-266.

Saito T, Murakami M, Shimazaki Y, Oobayashi K, Matsumoto S, Koga T (2003). Association between alveolar bone loss and elevated serum C-reactive protein in Japanese men. J Periodontol 74:1741-1746.

Saito T, Yamaguchi N, Shimazaki Y, Hayashida H, Yonemoto K, Doi Y, et al. (2008). Serum levels of resistin and adiponectin in women with periodontitis: the Hisayama study. J Dent Res 87:319-322.

Salcedo-Rocha AL, Sánchez-Mar M, López-Pérez ML, Preciado-Soltero HT, Figueroa-Suárez PA, Narváez-Aguirre VA, et al. (2006). Oral signs in patients with type-2 diabetes, hypertension and obesity. Rev Med Inst Mex Seguro Soc 44:203-210 [article in Spanish].

Schmidt AM, Weidman E, Lalla E, Yan SD, Hori O, Cao R, et al. (1996). Advanced glycation endproducts (AGEs) induce oxidant stress in the gingiva: a potential mechanism underlying accelerated periodontal disease associated with diabetes. $J$ Periodontal Res 31:508-515.

Semenkovich CF (2006). Insulin resistance and atherosclerosis. J Clin Invest 116:1813-1822.

Sharma P, Mishra S, Ajmera P, Mathur S (2005). Oxidative stress in metabolic syndrome. Indian J Clin Biochem 20:145-149.

Shimazaki Y, Saito T, Yonemoto K, Kiyohara Y, Iida M, Yamashita Y (2007). Relationship of metabolic syndrome to periodontal disease in Japanese women: the Hisayama Study. J Dent Res 86:271-275.

Sjogren P, Basu S, Rosell M, Silveira A, De Faire U, Vessby B, et al. (2005). Measures of oxidized low-density lipoprotein and oxidative stress are not related and not elevated in otherwise healthy men with the metabolic syndrome. Arterioscler Thromb Vasc Biol 25:2580-2586.

Soares AF, Guichardant M, Cozzone D, Bernoud-Hubac N, Bouzaïdi-Tiali $\mathrm{N}$, Lagarde M, et al. (2005). Effects of oxidative stress on adiponectin secretion and lactate production in 3T3-L1 adipocytes. Free Radic Biol Med 38:882-889.

Sonoki K, Nakashima S, Takata Y, Naito T, Fujisawa K, Ootsubo T, et al. (2006). Decreased lipid peroxidation following periodontal therapy in type 2 diabetic patients. J Periodontol 77:1907-1913.

Spiteller G (1998). Linoleic acid peroxidation - the dominant lipid peroxidation process in low density lipoprotein - and its relationship to chronic diseases. Chem Phys Lipids 95:105-162.

Spranger J, Kroke A, Möhlig M, Bergmann MM, Ristow M, Boeing H, et al. (2003). Adiponectin and protection against type 2 diabetes mellitus. Lancet 361:226-228; erratum in Lancet 361:1060, 2003.

Stewart JE, Wager KA, Friedlander AH, Zadeh HH (2001). The effect of periodontal treatment on glycemic control in patients with type 2 diabetes mellitus. J Clin Periodontol 28:306-310.

Suzuki K, Ito Y, Ochiai J, Kusuhara Y, Hashimoto S, Tokudome S, et al. (2003). Relationship between obesity and serum markers of oxidative stress and inflammation in Japanese. Asian Pac J Cancer Prev 4:259-266.

Takeda M, Ojima M, Yoshioka H, Inaba H, Kogo M, Shizukuishi S, et al. (2006). Relation of serum advanced glycation end products with deterioration of periodontitis in type 2 diabetic patients. $J$ Periodontol 77:15-20

Tilg H, Moschen AR (2008). Inflammatory mechanisms in the regulation of insulin resistance. Mol Med 14:222-231.

Tripathy D, Mohanty P, Dhindsa S, Syed T, Ghanim H, Aljada A, et al. (2003). Elevation of free fatty acids induces inflammation and impairs vascular reactivity in healthy subjects. Diabetes 52:2882-2887.

Trujillo ME, Scherer PE (2005). Adiponectin-journey from an adipocyte secretory protein to biomarker of the metabolic syndrome. J Intern Med 257:167-175.

Tsai C, Hayes C, Taylor GW (2002). Glycemic control of type 2 diabetes and severe periodontal disease in the US adult population. Community Dent Oral Epidemiol 30:182-192.

Türkoğlu O, Barış N, Kütükçüler N, Senarslan O, Güneri S, Atilla G (2008). Evaluation of serum anti-cardiolipin and oxidized low-density lipoprotein levels in chronic periodontitis patients with essential hypertension. J Periodontol 79:332-340.

Valentaviciene G, Paipaliene P, Nedzelskiene I, Zilinskas J, Anuseviciene OV (2006). The relationship between blood serum lipids and periodontal condition. Stomatologija 8:96-100.

Van Guilder GP, Hoetzer GL, Greiner JJ, Stauffer BL, DeSouza CA (2006). Influence of metabolic syndrome on biomarkers of oxidative stress and inflammation in obese adults. Obesity 14:2127-2131.

Wakai K, Kawamura T, Umemura O, Hara Y, Machida J, Anno T, et al. (1999). Associations of medical status and physical fitness with periodontal disease. J Clin Periodontol 26:664-672.

Wolk R, Berger P, Lennon RJ, Brilakis ES, Johnson BD, Somers VK (2004). Plasma leptin and prognosis in patients with established coronary atherosclerosis. J Am Coll Cardiol 44:1819-1824.

World Health Organization (1999). Definition, diagnosis and classification of diabetes mellitus and its complications: report of a WHO Consultation. Part 1. Diagnosis and classification of diabetes mellitus. http://www.diabetes.com.au/pdf/who_report.pdf. (Last accessed on 2008-11-19) 
World Health Organization (2005). The World Health Organization warns of the rising threat of heart disease and stroke as overweight and obesity rapidly increase. http://www.who.int/mediacentre/news/releases/2005/ pr44/en/index.html. (Last accessed 2008-11-19).

Wright E, Scism-Bacon JL, Glass LC (2006). Oxidative stress in type 2 diabetes: the role of fasting and postprandial glycemia. Int J Clin Pract 60:308-314.

Wu T, Trevisan M, Genco RJ, Falkner KL, Dorn JP, Sempos CT (2000). Examination of the relation between periodontal health status and cardiovascular risk factors: serum total and high density lipoprotein cholesterol, C-reactive protein, and plasma fibrinogen. Am J Epidemiol 151:273-282.

Yamaguchi N, Kukita T, Li YJ, Martinez Argueta JG, Saito T, Hanazawa S, et al. (2007). Adiponectin inhibits osteoclast formation stimulated by lipopolysaccharide from Actinobacillus actinomycetemcomitans. FEMS Immunol Med Microbiol 49:28-34.

Yamamoto Y, Hirose H, Saito I, Nishikai K, Saruta T (2004). Adiponectin, an adipocyte-derived protein, predicts future insulin resistance: twoyear follow-up study in Japanese population. J Clin Endocrinol Metab 89:87-90.

Yoon MS, Jankowski V, Montag S, Zidek W, Henning L, Schlüter H, et al. (2004). Characterisation of advanced glycation endproducts in saliva from patients with diabetes mellitus. Biochem Biophys Res Commun 323:377-381.

Zilinskas J, Zekonis J, Zekonis G, Valantiejiene A, Periokaite R (2007). The reduction of nitroblue tetrazolium by total blood in periodontitis patients and the aged. Stomatologija 9:105-108. 